\title{
Approximate hedging for non linear transaction costs on the volume of traded assets
}

\author{
Romuald Elie, Emmanuel Lépinette
}

\begin{abstract}
This paper is dedicated to the replication of a convex contingent claim $h\left(S_{1}\right)$ in a financial market with frictions, due to deterministic order books or regulatory constraints. The corresponding transaction costs rewrite as a non linear function $G$ of the volume of traded assets, with $G^{\prime}(0)>0$. For a stock with Black-Scholes mid-price dynamics, we exhibit an asymptotically convergent replicating portfolio, defined on a regular time grid with $n$ trading dates. Up to a well chosen regularization $h^{n}$ of the payoff function, we first introduce the frictionless replicating portfolio of $h^{n}\left(S_{1}^{n}\right)$, where $S^{n}$ is a fictive stock with enlarged local volatility dynamics. In the market with frictions, a proper modification of this portfolio strategy provides a terminal wealth, which converges in probability to the claim of interest $h\left(S_{1}\right)$, as $n$ goes to infinity. In terms of order book shapes, the exhibited replicating strategy only depends on the size $2 G^{\prime}(0)$ of the bid-ask spread. The main innovation of the paper is the introduction of a 'Leland type' strategy for non-vanishing (non-linear) transaction costs on the volume of traded shares, instead of the commonly considered traded amount of money. This induces lots of technicalities, that we pass through using an innovative approach based on the Malliavin calculus representation of the Greeks.
\end{abstract}

Key words Leland-Lott strategy, Delta hedging, Malliavin Calculus, transaction costs, order book.

Mathematics Subject Classification (2010) 91G20 ; 60G44 ; 60H07

\section{JEL Classification G11·G13}

\section{Introduction}

The current high frequency of trading on the financial markets does not allow to neglect the frictions induced by market orders for buying or selling a given number of shares. Depending on the liquidity of the stock of interest, the marginal price of 
any extra unit of stock can be significantly different. The shape of the order book and the size of the bid-ask spread determine the underlying cost induced by passing an order on the market. Modeling order book dynamics and more importantly quantifying the impact of the trades on the underlying price have brought a lot of attention in the recent literature. Our concern in this paper is to look towards efficient alternatives in order to replicate options in the presence of transaction costs, related to the presence of order books.

This kind of induced cost rewrites as a function of the traded amount of shares instead of the more classical and less realistic traded amount of money. For simplicity here, the order book shape is supposed to be deterministic and has a stationary asymptotic behavior when the number of traded shares goes to zero. More precisely, trading $\gamma$ shares of stock at time $t$ induces a cost $G(t, \gamma)$ where the possibly non-linear function $G$ satisfies $G(t, \gamma) \sim G^{\prime}(0)|\gamma|+O\left(|\gamma|^{2}\right)$, for $\gamma$ small enough. We consider a financial market with one bond normalized to 1 and one stock $S$ with Black-Scholes mid-price dynamics. Observe that $G^{\prime}(0)$ interprets as the half size of the bid-ask spread. The order book induces frictions on any position taken on the stock and we investigate the replication of a European option with payoff $h\left(S_{1}\right)$, where $h$ is a convex function.

In the classical framework of proportional transaction costs on the amount of traded money, Leland [8] introduced an ingenious method in order to hedge efficiently call options on a discrete time grid. His idea relies on the use of the frictionless hedging strategy associated to a Black-Scholes stock with a suitably enlarged volatility, related to the chosen frequency of trading. As the number of trading dates goes to infinity, Lott [10] or Kabanov and Safarian [6] verified that the terminal value of the corresponding portfolio converges to the claim $h\left(S_{1}\right)$ of interest, under the additional condition that the transaction costs coefficient vanishes sufficiently fast as well. This unrealistic assumption has recently been relieved by Lépinette [9] via a proper modification of the replicating strategy.

The main motivation of the paper is the introduction of 'Leland-Lott' approximate hedging strategies in the realistic framework described above, where the amount of transaction costs is a non linear function of the number of traded shares of asset. This particular feature implies that the natural 'Leland-type' enlarged volatility is associated to a local volatility model instead of a Black-Scholes one. Indeed, we consider the pricing function $\hat{C}^{n}$ and associated delta hedging strategy $\hat{C}_{x}^{n}$ induced by a fictive asset with local volatility

$$
\hat{\sigma}_{n}:(t, x) \mapsto \sqrt{|\sigma x|^{2}+\sigma G^{\prime}(0) \sqrt{\frac{8 n}{\pi}} x},
$$

where $\sigma$ is the Black-Scholes volatility of the stock and $1 / n$ is the mesh size of the regular revision grid.

In the imperfect market of interest, we exhibit a portfolio starting with initial wealth $\hat{C}^{n}\left(0, S_{0}\right)$ and induced by a proper modification of the delta hedging strategy $\left(\hat{C}_{x}^{n}\left(t, S_{t}\right)\right)_{0 \leq t \leq T}$, in the spirit of [9]. The main result of the paper is the convergence in probability of the terminal value of this portfolio to the claim of 
interest $h\left(S_{1}\right)$, as the number of revision dates $n$ tends to infinity. This convergence requires to consider payoff functions $h$ with bounded second derivatives. For derivatives with less regular payoff functions such as the classical call option, one simply needs to replace $h$ by a well chosen more regular payoff function $h^{n}$, characterized in terms of number of trading dates $n$ of the hedging strategy.

The approximate hedging strategy introduced in this paper allows therefore to replicate asymptotically a convex contingent claim $h\left(S_{1}\right)$ in a market with non vanishing transaction costs coefficient related to deterministic order books. The enhanced strategy only relies on the size $2 G^{\prime}(0)$ of the bid-ask spread and not on the global shape of the order book. The consideration of a fictive asset with local volatility dynamics of the form (1.1) induces lots of technicalities since the Lott-Kabanov methodology requires precise estimates on the sensitivities of the pricing function $\hat{C}^{n}$ in terms of the number $n$ of trading dates. The rather computational obtention of these estimates relies on an innovative approach based on the Malliavin representation of the Greeks introduced in [4].

The paper is organized as follows: The next section presents the financial market with frictions and the replication problem of interest. Section 3 is dedicated to the main results of the paper: the construction of the modified volatility and corresponding fictive pricing and hedging functions, the Delta correction for the consideration of non-vanishing transaction costs coefficient, the payoff regularization and the convergence of the enhanced replicating strategy. Section 4 details the proof of the convergence, whereas technical estimates on the derivatives of the fictive pricing function $\hat{C}^{n}$ are reported in Section 5 .

Notations. For a function $f$ from $[0,1] \times \mathbb{R}$ to $\mathbb{R}$, we denote by $f_{t}, f_{x}, f_{t x}, f_{x x}$, ... the time and space partial derivatives. For a function $f$ from $\mathbb{R}$ to $\mathbb{R}$, the first and second derivatives are simply denoted $\nabla f$ and $\nabla^{2} f$. We denote by $C$ a generic constant, which may vary from line to line. For possibly random constants, we use the notation $C_{\omega}$.

\section{Hedging under transaction costs on the traded volume of shares}

In this section, we introduce the market model and formulate the financial derivative replication problem under transaction costs induced by order book frictions.

\subsection{The market model}

We consider a financial market defined on a probability space $(\Omega, \mathcal{F}, \mathbb{Q})$, endowed with a 1-dimensional Brownian motion $W$. We denote by $\mathbb{F}=\left(\mathcal{F}_{t}\right)_{t \geq 0}$ the completion of the filtration generated by $W$.

Our model is the standard two-asset model with the time horizon $T=1$ assuming that it is specified under the unique martingale measure $\mathbb{Q}$. The non-risky asset is the numéraire $S^{0}=1$, and the dynamics of the risky asset is given by the 
stochastic equation

$$
S_{t}=S_{0}+\int_{0}^{t} \sigma S_{u} d W_{u}, \quad 0 \leq t \leq 1
$$

where $\sigma>0$ is a constant. Up to considering discounted processes, all the results of the paper extend as usual to financial markets with non zero deterministic interest rates.

In a frictionless complete market of this form, the price at time $t$ of a financial derivative $h\left(S_{1}\right)$ is given by $C\left(t, S_{t}\right)$ where $C$ is the unique solution of the PDE

$$
\left(\mathbf{e}_{\mathbf{0}}\right)=\left\{\begin{array}{l}
C_{t}(t, x)+\frac{1}{2} \sigma^{2} x^{2} C_{x x}(t, x)=0, \quad(t, x) \in[0,1) \times(0, \infty) \\
C(1, x)=h(x), \quad x \in(0, \infty)
\end{array} .\right.
$$

In presence of realistic transaction costs, where continuous hedging is not adequate anymore, this paper develops an asymptotic hedging strategy for the financial derivative $h\left(S_{1}\right)$.

\subsection{The order book frictions}

We intend to take into account the frictions induced by the use of market orders in the financial market. When a portfolio manager buys or sells a given quantity $\gamma \neq 0$ of stock $S$, the presence of order books implies an additional cost, which is related to the volume $\gamma$ of the order. We model these order book related costs via the introduction of a non linear continuous deterministic cost function $G$. Whenever an agent trades a (possibly negative) quantity $\gamma$ of stocks $S$ on the financial market at time $t$, he shall pay an immediate cost $G(t, \gamma)>0$.

We make the following stationary assumption on the asymptotic behavior of the cost function $G$ on the neighborhood of $\gamma=0$.

Condition (G): There exists a constant $G^{\prime}(0)>0$ such that

$$
G(t, \gamma)=G^{\prime}(0)|\gamma|+O\left(|\gamma|^{2}\right), \quad 0 \leq t \leq 1
$$

Remark 2.1 When $S$ represents the mid-price dynamics of the risky financial asset, $2 G^{\prime}(0)$ interprets simply as the bid-ask spread of the asset in the order book of interest. We shall see in the following that for asymptotic replication purpose, only the size $2 G^{\prime}(0)$ of the bid-ask spread is relevant in our approach.

Remark 2.2 Of course, assuming that the order book is deterministic and that the bid-ask spread remains constant is unrealistic and hence restrictive. Nevertheless, we outline in this paper that this simple framework already raises interesting mathematical problems and leads to promising conclusions. The consideration of dynamic random order books, for which no unanimous model has emerged in the literature, shall be left for further research. 


\subsection{Portfolio dynamics and replication}

Due to the presence of frictions on the market, inducing direct or indirect transaction costs, we only consider portfolio strategies, where the manager changes his market position on a finite number $n$ of revision dates $\left(t_{i}^{n}\right)_{0 \leq i \leq n}$. For simplicity, we assume in the paper that the revision dates $\left(t_{i}^{n}\right)$ define a uniform deterministic time grid, i.e. $t_{i}^{n}:=i / n$, for $0 \leq i \leq n$.

Remark 2.3 As observed in [2] or [13], the use of non uniform time grid, where the number of trading dates increases as the maturity is getting closer, allows to improve the convergence of the Leland type approximate hedging strategy. One can expect this property to remain satisfied in our context. A rigorous proof of this result requires very computational finer estimates, which go beyond the scope of this (already technical) paper. For the consideration of random time nets, we refer to the nice results of [5], which produces a robust asymptotic hedging strategy for vanishing linear transaction costs written in terms of the traded amount of money.

A portfolio on the time interval $[0,1]$ is given by an initial capital $x \in \mathbb{R}$ and an $\mathcal{F}$-adapted piecewise-constant process $\left(H^{n}\right)_{n \in \mathbb{N}}$, where $H_{t_{i}^{n}}^{n} \in \mathcal{L}^{2}(\Omega)$ represents the number of shares of stock hold in the portfolio on the time interval $\left[t_{i}^{n}, t_{i+1}^{n}\right)$, for any $0 \leq i<n$. Due to the order book frictions, the value of the portfolio process $V^{n}$ associated to the piecewise-constant investment strategy $H^{n}$ is given by

$$
V_{t}^{n}=V_{0}^{n}+\int_{0}^{t} H_{u}^{n} d S_{u}-\sum_{t_{i}^{n} \leq t} G\left(t_{i}^{n}, H_{t_{i}^{n}}^{n}-H_{t_{i-1}^{n}}^{n}\right), \quad 0 \leq t \leq 1, \quad n \in \mathbb{N} .
$$

We aim at hedging the contingent claim with payoff $h\left(S_{1}\right)$, where $h$ is a convex function, for which precise regularity requirements are given in Section 3.3 below. We look towards a portfolio $V^{n}$, with terminal value converging to $h\left(S_{1}\right)$ as the number of trading dates $n$ tends to infinity.

\section{Asymptotic hedging via volatility modification and payoff regularization}

In order to exhibit a portfolio strategy, whose asymptotic terminal value attains the claim of interest $h\left(S_{1}\right)$ despite the frictions, we formally explain in Section 3.1 the Leland methodology and consider a fictive asset with upgraded volatility. Since transaction costs rewrite in our framework as a function of the volume of traded asset, the fictive asset has non Lipschitz local volatility dynamics. After verifying in Section 3.2 that this stochastic differential equation has a unique solution, we introduce the corresponding pricing and hedging functions of the claim $h\left(S_{1}\right)$ for a frictionless market. Up to a proper strategy modification, we exhibit in Section 3.4 an asymptotic hedging strategy for the convex claim $h\left(S_{1}\right)$. For payoff functions with few regularity such as call option, a well chosen additional regularization method is exposed in Section 3.3.

\subsection{Construction of the enlarged volatility function}

In the frictionless Black-Scholes model, the price function of the convex claim $h\left(S_{1}\right)$ is the unique solution $C(.,$.$) of the PDE \left(\mathbf{e}_{\mathbf{0}}\right)$ and the exact self-financing 
replication portfolio is given by

$$
C\left(t, S_{t}\right)=\mathbb{E} h\left(S_{1}\right)+\int_{0}^{t} C_{x}\left(u, S_{u}\right) d S_{u}, \quad 0 \leq t \leq 1 .
$$

It exactly replicates the contingent claim $h\left(S_{1}\right)$ and is self-financing. In the presence of transaction costs, Leland suggested in his famous paper [8] to substitute the volatility $\sigma$ by an artificially enlarged one $\widehat{\sigma}_{n}$, related to the mesh $1 / n$ of the trading replication grid. We briefly recall the main ideas behind this volatility enlargement and detail formally how it adapts to the framework of frictions considered here.

For a sequence of volatility functions $\left(\widehat{\sigma}_{n}\right)_{n}$ to be determined below, consider the following PDEs

$$
\left\{\begin{array}{l}
u_{t}(t, x)+\frac{1}{2} \widehat{\sigma}_{n}^{2}(x) x^{2} u_{x x}(t, x)=0, \quad(t, x) \in[0,1) \times(0, \infty) \\
u(1, x)=h(x), \quad x \in(0, \infty)
\end{array},\right.
$$

for $n \in \mathbb{N}$. The solution $C^{n}$ of this equation (if it exists) is the frictionless pricing function of a financial derivative with payoff function $h$, whenever the stock has $\widehat{\sigma}^{n}$ local volatility dynamics.

We look towards a volatility function $\widehat{\sigma}^{n}$ allowing to take into account the transaction costs induced on the $n$ trading dates. More precisely, Ito's formula implies that the formally supposed smooth function $C^{n}$ verifies

$$
C^{n}\left(t, S_{t}\right)=C^{n}\left(0, S_{0}\right)+\int_{0}^{t} C_{x}^{n}\left(u, S_{u}\right) d S_{u}+\frac{1}{2} \int_{0}^{t}\left[\sigma^{2}-\widehat{\sigma}_{n}^{2}\left(S_{u}\right)\right] S_{u}^{2} C_{x x}^{n}\left(u, S_{u}\right) d u,
$$

for $0 \leq t \leq 1$ and $n \in \mathbb{N}$. Hence, the process $\left(C^{n}\left(t, S_{t}\right)\right)_{0 \leq t \leq 1}$ can be approximately identified as a portfolio process with dynamics of the form (2.2) whenever the last term on the right hand side above corresponds to the transaction costs cumulative sum, i.e. equalizing the variations:

$$
\frac{1}{2}\left[\sigma^{2}-\widehat{\sigma}_{n}^{2}\left(S_{u}\right)\right] S_{u}^{2} C_{x x}^{n}\left(u, S_{u}\right) \Delta u \simeq-G\left(u, C_{x}^{n}\left(u+\Delta u, S_{u+\Delta u}\right)-C_{x}^{n}\left(u, S_{u}\right)\right),
$$

for $n \in \mathbb{N}$. A formal Taylor approximation gives

$$
\begin{aligned}
C_{x}^{n}\left(u+\Delta u, S_{u+\Delta u}\right)-C_{x}^{n}\left(u, S_{u}\right) & =C_{x t}^{n}\left(u, S_{u}\right) \Delta u+C_{x x}^{n}\left(u, S_{u}\right)\left(S_{u+\Delta u}-S_{u}\right), \\
& \simeq C_{x x}^{n}\left(u, S_{u}\right)\left(S_{u+\Delta u}-S_{u}\right),
\end{aligned}
$$

for $n \in \mathbb{N}$. Since $h$ is a convex function, we expect $C_{x x}^{n} \geq 0$ and it follows formally from Condition (G) together with the relation $S_{u+\Delta u}-S_{u} \simeq \sigma S_{u}\left(W_{u+\Delta u}-W_{u}\right)$ that

$$
\frac{1}{2}\left[\sigma^{2}-\widehat{\sigma}_{n}^{2}\left(S_{u}\right)\right] \Delta u \simeq-G^{\prime}(0) \sigma\left|W_{u+\Delta u}-W_{u}\right| \frac{1}{S_{u}}, \quad n \in \mathbb{N} .
$$

Taking the conditional expectation given $\mathcal{F}_{u}$ and plugging the classical estimate $E\left|W_{u+\Delta u}-W_{u}\right|=\sqrt{2 \Delta u / \pi}$, this leads to

$$
\frac{1}{2}\left[\sigma^{2}-\widehat{\sigma}_{n}^{2}\left(S_{u}\right)\right] \Delta u \simeq-G^{\prime}(0) \frac{\sigma}{S_{u}} \sqrt{\frac{2 \Delta u}{\pi}}, \quad n \in \mathbb{N} .
$$


For the regular trading grid considered here, $\Delta u=1 / n$ provides the following candidate for the upgraded volatility function:

$$
\widehat{\sigma}_{n}^{2}: x \in(0, \infty) \mapsto \sigma^{2}+G^{\prime}(0) n^{1 / 2} \sqrt{\frac{8}{\pi}} \frac{\sigma}{x}, \quad n \in \mathbb{N}
$$

Observe that this candidate upgraded local volatility function is degenerate at 0 and we prove in the next paragraph the well posed-ness of the corresponding local volatility fictive asset and associated pricing function.

3.2 The fictive asset dynamics

Let us consider a sequence of fictive assets, whose dynamics are given by the candidate upgraded volatility $\left(\widehat{\sigma}_{n}\right)$ defined in (3.3). We expect the fictive assets $\left(\widehat{S}^{n}\right)_{n}$ to solve the following stochastic differential equation

$$
\widehat{S}_{t}^{n}=S_{0}+\int_{0}^{t} \widehat{\gamma}_{n}\left(\widehat{S}_{u}^{n}\right) d W_{u}, \quad 0 \leq t \leq T, \quad n \in \mathbb{N},
$$

where we introduced the notation

$$
\widehat{\gamma}_{n}: x \mapsto \widehat{\sigma}_{n}(x) x=\sqrt{\sigma^{2} x^{2}+\sigma \gamma_{n}|x|}, \quad \text { with } \quad \gamma_{n}:=G^{\prime}(0) n^{1 / 2} \sqrt{\frac{8}{\pi}}, \quad n \in \mathbb{N} .
$$

Since the diffusion coefficients $\left(\widehat{\gamma}_{n}\right)$ are not Lipschitz, the existence of a unique process with such dynamics does not follow from the classical theorems. We puzzle out this difficulty using the Engelbert \& Schmidt criterion as detailed in the following lemma.

Lemma 3.1 Whatever initial condition $(t, x) \in[0,1] \times(0, \infty)$, the stochastic differential equation (3.4) admits a unique strong solution $\left(\widehat{S}_{s}^{n}\right)_{t \leq s \leq 1}$, starting from $x$ at time t. Furthermore, this solution remains non-negative.

Proof. We fix $n \in \mathbb{N}$ and $(t, x) \in[0,1] \times(0, \infty)$. For any $z \in \mathbb{R}$, observe that the diffusion coefficient $\widehat{\gamma}_{n}$ defined in (3.5) satisfies:

$$
\text { if } \int_{-\varepsilon}^{\varepsilon} \frac{d y}{\left|\widehat{\gamma}_{n}(z+y)\right|^{2}}=\infty, \quad \text { for any } \varepsilon>0, \quad \text { then } \quad \widehat{\gamma}_{n}(z)=0 .
$$

Indeed, for $z \neq 0$, taking $\varepsilon=|z| / 2$, we get $\int_{-\varepsilon}^{\varepsilon} \frac{d y}{\left|\widehat{\gamma}_{n}(z+y)\right|^{2}}<\infty$, so that the left hand side condition of (3.6) implies $z=0$, leading to $\widehat{\gamma}_{n}(z)=0$. Hence, the diffusion coefficient $\widehat{\gamma}_{n}$ satisfies the Engelbert \& Schmidt criterion, and, there exists a weak solution to (3.4) with initial condition $(t, x)$, see Theorem 5.4 in Section 5 of [7].

We now observe that the diffusion coefficient $\widehat{\gamma}_{n}$ also satisfies

$$
\begin{aligned}
\left|\widehat{\gamma}_{n}(z)-\widehat{\gamma}_{n}(y)\right| & =\left|\sqrt{\sigma^{2} z^{2}+\sigma \gamma_{n}|z|}-\sqrt{\sigma^{2} y^{2}+\sigma \gamma_{n}|y|}\right| \\
& \leq \sigma|z-y|+\left|\sqrt{\sigma^{2} y^{2}+\sigma \gamma_{n}|z|}-\sqrt{\sigma^{2} y^{2}+\sigma \gamma_{n}|y|}\right|, \quad(z, y) \in \mathbb{R}^{2},
\end{aligned}
$$


since the derivative of $y \mapsto \sqrt{\sigma^{2} y^{2}+\sigma \gamma_{n}|z|}$ is upper bounded by $\sigma$. We deduce

$$
\left|\widehat{\gamma}_{n}(z)-\widehat{\gamma}_{n}(y)\right| \leq \sigma|z-y|+\sqrt{\sigma \gamma_{n}} \sqrt{|| z|-| y||} \leq \ell(|z-y|), \quad(z, y) \in \mathbb{R}^{2},
$$

with $\ell: u \mapsto \sigma u+\sqrt{\sigma \gamma_{n} u}$. Since $\int_{0}^{\varepsilon} \frac{d u}{|\ell(u)|^{2}}=\infty$, for any $\varepsilon>0$, we deduce from Proposition 2.13 in Section 5 of [7] that pathwise uniqueness holds for the stochastic differential equation (3.1). Together with the existence of a weak solution verified above, this implies the existence of a unique strong solution to (3.1) for any initial condition $(t, x)$, see Corollary 3.23 in Section 5 of [7].

Finally, $\widehat{S}^{n}$ remains non-negative, since it is continuous and Markovian, and the unique strong solution starting at 0 is the null one.

\subsection{Payoff regularization and related pricing function}

We now inquire the properties of the pricing functions associated to the fictive assets $\left(\widehat{S}^{n}\right)_{n}$ and first discuss the regularity of the payoff function of interest.

We aim at hedging the contingent claim with payoff $h\left(S_{1}\right)$, where the payoff function $h$ is supposed to satisfy the following:

Condition (P): The convex function $h:[0, \infty) \rightarrow \mathbb{R}$ is affine outside the interval $[1 / K, K]$, with $K>1$.

Observe that most of the classical convex payoffs satisfy this condition. In particular, under Condition (P), the map $h$ is Lipschitz and we denote by $L>0$ its smallest Lipschitz constant.

In the following, we shall sometimes require the payoff function to be continuously differentiable. Besides, in order to consider non-vanishing transaction costs, we need a control on the second order variations of the payoff function. In order to do so, we regularize the convex map $h$, as detailed in the following lemma.

Lemma 3.2 There exists a sequence of convex maps $\left(h^{n}\right)_{n}$ valued in $C^{2}([0, \infty), \mathbb{R})$ such that, for $n$ large enough,

$$
\left\|h^{n}-h\right\|_{\infty} \leq L \frac{\ln (n)}{\gamma_{n}^{1 / 6}}, \quad\left\|\nabla h^{n}\right\|_{\infty} \leq L, \quad\left\|\nabla^{2} h^{n}\right\|_{\infty} \leq 3 L \frac{\gamma_{n}^{1 / 6}}{\ln (n)} \mathbf{1}_{[1 / 2 K, 2 K]} .
$$

Proof. We observe that $h$ is affine on $[0,1 / K]$ and introduce the extension of $h$ on $\mathbb{R}$, which remains affine with the same slope on $(-\infty, 0)$. For simplicity, this extended map is also denoted $h$. For $n \in \mathbb{N}$, we introduce the convolution between $h$ and the square kernel with support $\left[-\ln (n) / \gamma_{n}^{1 / 6}, \ln (n) / \gamma_{n}^{1 / 6}\right]$ :

$$
h^{n}: x \in[0, \infty) \mapsto \frac{4}{3} \int_{-1}^{1} h\left(x+\frac{y \ln (n)}{\gamma_{n}^{1 / 6}}\right)\left(1-y^{2}\right) d y .
$$

Since $h$ is $L$-Lipschitz and $\int_{-1}^{1}\left(1-y^{2}\right) d y=3 / 4$, we compute

$$
\left\|h^{n}-h\right\|_{\infty} \leq \frac{4}{3} \int_{-1}^{1}\left|\frac{L \ln (n) y}{\gamma_{n}^{1 / 6}}\right|\left(1-y^{2}\right) d y=\frac{2 L}{3} \frac{\ln (n)}{\gamma_{n}^{1 / 6}} \leq L \frac{\ln (n)}{\gamma_{n}^{1 / 6}}, \quad n \in \mathbb{N} .
$$


Fix $n \in \mathbb{N}$. Observe that $h^{n} \in C^{2}([0, \infty), \mathbb{R})$ and, denoting abusively $\nabla h$ the right derivative of $h$, we have

$$
\begin{aligned}
\nabla h^{n}(x) & =\frac{4}{3} \int_{-1}^{1} \nabla h\left(x+\frac{y \ln (n)}{\gamma_{n}^{1 / 6}}\right)\left(1-y^{2}\right) d y \\
& =\frac{4}{3} \int_{x-\frac{1}{n}}^{x+\frac{1}{n}} \nabla h(z)\left(1-\left|\frac{\gamma_{n}^{1 / 6}}{\ln (n)}(z-x)\right|^{2}\right) \frac{\gamma_{n}^{1 / 6}}{\ln (n)} d z, \quad x \geq 0 .
\end{aligned}
$$

Since $\|\nabla h\|_{\infty} \leq L$, we deduce that $\left\|\nabla h^{n}\right\|_{\infty} \leq L$.

Differentiating the second expression of $\nabla h^{n}$ above, we deduce that

$$
\nabla^{2} h^{n}(x)=\frac{4}{3} \int_{x-\frac{1}{n}}^{x+\frac{1}{n}} \nabla h(z) \frac{2 \gamma_{n}^{2 / 6}}{\ln (n)^{2}}(x-z) \frac{\gamma_{n}^{1 / 6}}{\ln (n)} d z=\frac{8}{3} \int_{-1}^{1}-\nabla h\left(x+\frac{y \ln (n)}{\gamma_{n}^{1 / 6}}\right) \frac{\gamma_{n}^{1 / 6}}{\ln (n)} y d y,
$$

for $x \geq 0$. Using once again that $\|\nabla h\|_{\infty} \leq L$, this yields

$$
\left\|\nabla^{2} h^{n}\right\|_{\infty} \leq \frac{8 L}{3} \int_{-1}^{1}|y| \frac{\gamma_{n}^{1 / 6}}{\ln (n)} d y=\frac{8 L}{3} \frac{\gamma_{n}^{1 / 6}}{\ln (n)} \leq 3 L \frac{\gamma_{n}^{1 / 6}}{\ln (n)} .
$$

Besides, since $h$ is affine on $[K, \infty)$, we deduce that

$$
h^{n}(x)=\frac{4}{3} \int_{-1}^{1} \nabla h(K)\left(x-K+\frac{y \ln (n)}{\gamma_{n}^{1 / 6}}\right)\left(1-y^{2}\right) d y=\nabla h(K)(x-K)=h(x),
$$

for any $x \geq K+\ln (n) / \gamma_{n}^{1 / 6}$. The exact same reasoning applies for $x \leq 1 / K-$ $\ln (n) / \gamma_{n}^{1 / 6}$. Hence, for $n$ large enough such that $\gamma_{n}^{1 / 6} / \ln (n) \geq K, h^{n}$ is affine and therefore $\nabla^{2} h^{n}=0$ outside the interval $[1 / 2 K, 2 K]$. Combined with (3.8), this completes the proof.

Remark 3.4 Whenever $h$ is valued in $C^{2}([0, \infty), \mathbb{R})$, the regularization procedure is not necessary since (3.7) is satisfied as soon as $n$ is large enough. Hence one can simply use $h$ instead of $\left(h^{n}\right)_{n}$.

The sequence of regularized approximating payoff functions $\left(h^{n}\right)_{n}$ in hand, we can now introduce the associated valuation PDEs, given by:

$$
\left(\mathbf{e}_{\mathbf{n}}\right)=\left\{\begin{array}{l}
\widehat{C}_{t}^{n}(t, x)+\frac{1}{2} \widehat{\sigma}_{n}^{2}(x) x^{2} \widehat{C}_{x x}^{n}(t, x)=0, \quad(t, x) \in[0,1) \times(0, \infty), \\
\widehat{C}^{n}(1, x)=h^{n}(x), \quad x \in(0, \infty) .
\end{array}\right.
$$

for $n \in \mathbb{N}$. The existence of a unique strong solution for this PDE is given in Proposition 3.3 below. For sake of completeness and since the corresponding differential operator is not uniformly parabolic on $[0,1) \times(0, \infty)$, the proof of this proposition is reported in Appendix. As expected, the solution of the PDE interprets as the valuation function of the option with payoff $h^{n}$ on the terminal value of the fictive asset $\widehat{S}_{1}^{n}$, introduced in the previous section.

Proposition 3.3 For any $n \in \mathbb{N}$, the PDE $\left(\mathbf{e}_{\mathbf{n}}\right)$ has a unique solution denoted $\widehat{C}^{n}$, which moreover satisfies

$$
\widehat{C}^{n}(t, x)=\mathbb{E}_{t, x}\left[h^{n}\left(\widehat{S}_{1}^{n}\right)\right], \quad(t, x) \in[0,1] \times(0, \infty), \quad n \in \mathbb{N} .
$$


3.4 Delta correction and asymptotic hedging for non vanishing transaction costs coefficient

Even in a frictionless complete setting, a contingent claim can never be perfectly replicated in practice, since continuous time hedging is not feasible. As detailed in Section 2.3, we consider portfolios where the position in the assets changes on the regular discrete time grid $\left(t_{i}^{n}\right)_{i \leq n}$. In this framework, we claim that the upgrade $\left(\widehat{\sigma}_{n}\right)_{n}$ of volatility and the regularization $\left(h^{n}\right)_{n}$ of the payoff detailed in Section 3.2 and Section 3.3 allows to counterbalance asymptotically the frictions due to order book related transaction costs. This claim is the content of the next theorem, which is the main result of the paper.

Theorem 3.4 Consider the sequence of portfolios $\left(V^{n}\right)_{n}$ associated to the initial conditions $\left(\widehat{C}^{n}\left(0, S_{0}\right)\right)_{n}$ and the investment strategies $\left(H^{n}\right)_{n}$ defined by

$$
H_{t}^{n}:=\widehat{C}_{x}^{n}\left(t_{i}^{n}, S_{t_{i}^{n}}\right)-\sum_{j \leq i}\left(\widehat{C}_{x}^{n}\left(t_{j}^{n}, S_{t_{j-1}^{n}}\right)-\widehat{C}_{x}^{n}\left(t_{j-1}^{n}, S_{t_{j-1}^{n}}\right)\right),
$$

for $t \in\left[t_{i}^{n}, t_{i+1}^{n}\right)$ and $0 \leq i<n$. Then, the sequence of portfolio values rewrite

$$
V_{t}^{n}=\widehat{C}^{n}\left(0, S_{0}\right)+\int_{0}^{t} H_{u}^{n} d S_{u}-\sum_{t_{i}^{n} \leq t} G\left(t_{i}^{n}, H_{t_{i}^{n}}^{n}-H_{t_{i-1}^{n}}^{n}\right), \quad 0 \leq t \leq 1, \quad n \in \mathbb{N},
$$

and $\left(V_{1}^{n}\right)_{n}$ converges in probability to the payoff $h\left(S_{1}\right)$ as $n$ goes to $\infty$.

The proof of this theorem is presented in Section 4 below, and it requires sharp estimates on the derivatives of $\left(\widehat{C}^{n}\right)_{n}$, whose proofs are postponed to Section 5 .

Remark 3.5 Observe that the hedging strategy does not simply consist in considering the Delta associated to the fictive asset $\left(\widehat{S}^{n}\right)_{n}$. Indeed, as observed in $[6,11]$ for the classical framework of transaction costs proportional to the amount of money, this original Leland replicating strategy does not converge to the claim of interest, unless the transaction costs vanish fast enough as the number of trading dates $n$ increases. As in [9], the extra term in the definition of $\left(H_{n}\right)_{n}$ allows to consider non vanishing transaction costs. In particular, observe that the change of position at time $t_{i}^{n}$, for $i \leq n$, in the portfolio $V^{n}$ is given by $\widehat{C}_{x}^{n}\left(t_{i}^{n}, S_{t_{i}^{n}}\right)-\widehat{C}_{x}^{n}\left(t_{i}^{n}, S_{t_{i-1}^{n}}\right)$.

Remark 3.6 Our main result also allows to quantify the effects of a volume based trading taxation, on the cost of hedging strategies for convex derivatives. Indeed, in order to render most of the high frequency trading arbitrage opportunities irrelevant, the regulator is still looking towards the best way to create a tax on trading orders. Nevertheless, the exact consequences of such a regulation on asset management strategies or more generally risk management strategies is not yet completely understood. Simple questions on this subject still lack fully satisfying answers: Should the regulator create a tax on the volume of traded asset or the quantity of traded money? Should he use a linear tax? What are the consequences of using a different shape of tax function? In our simplifying Black-Scholes framework, our conclusions are that the global shape of the taxation does not really matters from a hedging perspective since only the asymptotic behavior around 0 is relevant. Besides, Theorem 3.4 exhibits the volatility change related to a volume based taxation instead of a more classical amount based one. 


\section{Proof of the main result}

Due to the consideration of volume related non linear transaction costs, the exhibited trading strategy is based on a pricing function of a stock model with non linear dynamics. Hence, classical estimates are not available for the sensitivities of the price function in terms of the volatility parameter. But, we require to understand precisely the dependence of the price sensitivities with respect to the number of trading dates $n$ which affects the modified volatility parameter. We overcome this difficulty, using Malliavin derivative type representation of the Greeks, as detailed in the next subsection. This leads to sharp estimates, which allow to derive the convergence of the approximating replicating portfolio to the claim of interest at maturity.

4.1 Representation and estimates for the modified price function sensitivities

Recall that the price function $\hat{C}^{n}$ is given by

$$
\hat{C}^{n}:(t, x) \mapsto \mathbb{E}_{t, x}\left[h^{n}\left(\widehat{S}_{1}^{n}\right)\right] .
$$

A well chosen probability change leads classically to a nice representation of the Delta of the option presented below.

Lemma 4.1 For $n \in \mathbb{N}$ and any initial condition $(t, x) \in[0,1] \times(0, \infty)$, the s.d.e.

$$
d \widetilde{S}_{u}^{n}=\widehat{\gamma}_{n}\left(\widetilde{S}_{u}^{n}\right) d W_{u}+\widehat{\gamma}_{n} \nabla \widehat{\gamma}_{n}\left(\widetilde{S}_{u}^{n}\right) d u
$$

has a unique solution $\widetilde{S}^{n}$, which moreover remains strictly positive. Besides, we have

$$
\widehat{C}_{x}^{n}(t, x)=\mathbb{E}_{t, x}\left[\nabla h^{n}\left(\widetilde{S}_{1}^{n}\right)\right], \quad(t, x) \in[0,1] \times(0, \infty), \quad n \in \mathbb{N} .
$$

Proof. Fix $n \in \mathbb{N}$. The existence of a unique solution to (4.12) follows from similar arguments as the one presented in Lemma 3.1. Besides, since $\int_{0}^{1} \rho_{n}(u) d u=\infty$ where

$$
\rho_{n}: u \mapsto \exp \left\{\int_{u}^{1} \frac{2 \sigma^{2} y+\sigma \gamma_{n}}{\sigma^{2} y^{2}+\sigma \gamma_{n} y} d y\right\}=\frac{\sigma^{2}+\sigma \gamma_{n}}{\sigma^{2} u^{2}+\sigma \gamma_{n} u},
$$

Theorem 2.16 and 2.17 , [1], ensure that $\widetilde{S}^{n}$ remains strictly positive for a given positive initial condition.

The mappings $y \mapsto \widehat{\sigma}^{n}\left(e^{y}\right)$ and $y \mapsto\left|\widehat{\sigma}^{n}\left(e^{y}\right)\right|^{2}$ admit locally Lipschitz first derivatives because their second derivatives are locally bounded. Let denote $\bar{S}^{n}:=$ $\ln \widehat{S}^{n}$. By virtue of Theorem 39 (V.7) and Theorem 38 (V.7)[12], we deduce that there exists a version of the mapping $y \mapsto \bar{S}_{t, y}^{n}$, which is continuously differentiable and so is $x \mapsto \widehat{S}_{t, x}^{n}$ on $(0, \infty)$, for any $t \in(0,1)$. Precisely, for a given initial condition $(t, x) \in[0,1] \times(0, \infty)$, the tangent process $\nabla \widehat{S}^{n}$ is given by

$$
\nabla \widehat{S}_{u}^{n}=1+\int_{t}^{u} \nabla \widehat{\gamma}_{n}\left(\widehat{S}_{s}^{n}\right) \nabla \widehat{S}_{s}^{n} d W_{s}, \quad t \leq s \leq T .
$$


Besides, differentiating expression (4.12) provides $\widehat{C}_{x}^{n}(t, x)=\mathbb{E}_{t, x}\left[\nabla h^{n}\left(\widehat{S}_{1}^{n}\right) \nabla \widehat{S}_{1}^{n}\right]$. Assume for the moment that $\nabla \widehat{S}^{n}$ is a positive martingale and introduce the new equivalent probability $\mathbb{P}^{n}$ defined by $d \mathbb{P}^{n}=\nabla \widehat{S}_{1}^{n} d \mathbb{Q}$, so that

$$
\widehat{C}_{x}^{n}(t, x)=\mathbb{E}_{t, x}^{\mathbb{P}^{n}}\left[\nabla h^{n}\left(\widehat{S}_{1}^{n}\right)\right] .
$$

Girsanov theorem asserts that the process $W^{n}$ given by $d W_{u}^{n}=d W_{u}-\nabla \widehat{\gamma}^{n}\left(\widehat{S}_{u}^{n}\right) d u$ is a standard Brownian motion under $\mathbb{P}^{n}$. Hence, the dynamics of $\widehat{S}^{n}$ under $\mathbb{P}^{n}$ are given by

$$
d \widehat{S}_{u}^{n}=\widehat{\gamma}^{n}\left(\widehat{S}_{u}^{n}\right) d W_{u}^{n}+\widehat{\gamma}^{n} \nabla \widehat{\gamma}^{n}\left(\widehat{S}_{u}^{n}\right) d u .
$$

Therefore, the law of $\widehat{S}^{n}$ under $\mathbb{P}^{n}$ is identical to the one of $\widetilde{S}^{n}$ under $\mathbb{Q}$ and (4.14) rewrites as (4.13).

The rest of the proof is dedicated to the verification that $\nabla \widehat{S}^{n}$ is indeed a positive martingale.

For any $p \in \mathbb{N}$, let us introduce the stopping time

$$
\tau^{p}:=\inf \left\{s \leq 1: \widehat{S}_{s}^{n} \leq x /(1+p)\right\},
$$

with the convention that $\inf \emptyset=\infty$. Applying Gronwall's lemma, we verify that $\sup _{t \leq s \leq 1} \nabla \widehat{S}_{s \wedge \tau^{n}}^{n}$ is square integrable, hence $\nabla \widehat{S}_{. \wedge \tau^{n}}^{n}$ is a martingale. Let us define the change of measure $d \mathbb{Q}^{p}:=\nabla \widehat{S}_{1 \wedge \tau^{p}} d \mathbb{Q}$. Then,

$$
\mathbb{E}\left[\nabla \widehat{S}_{1}^{n}\right] \geq \mathbb{E}\left[\nabla \widehat{S}_{1 \wedge \tau^{p}}^{n} 1_{\tau^{p}=\infty}\right]=\mathbb{Q}^{p}\left(\tau^{p}=\infty\right), \quad p \in \mathbb{N} .
$$

As $\left(\tau^{p}\right)_{p}$, let us define the sequence $\left(\tilde{\tau}^{p}\right)_{p}$ associated to the process $\widetilde{S}^{n}$ given by (4.12). By construction, observe that $\tau^{p}$ has the same law under $\mathbb{Q}^{p}$ than $\widetilde{\tau}^{p}$ under $\mathbb{Q}$, for any $p \in \mathbb{N}$. It follows that $\mathbb{Q}^{p}\left(\tau^{p}=\infty\right)=\mathbb{Q}\left(\tilde{\tau}^{p}=\infty\right) \rightarrow \mathbb{Q}\left(\tilde{\tau}^{\infty}=\infty\right)$ where $\tilde{\tau}^{\infty}$ is the first time when $\widetilde{S}^{n}$ hits zero. But $\widetilde{S}^{n}$ remains strictly positive, so that (4.15) implies that $\mathbb{E}\left[\nabla \widehat{S}_{1}^{n}\right] \geq 1$. Since $\nabla \widehat{S}^{n}$ is a supermartingale, we then conclude.

We now provide an expression for the second derivative of the price function $\hat{C}^{n}$, in the spirit of the Malliavin representation of the Greeks presented in [4].

Lemma 4.2 For any $n \in \mathbb{N}$, we have

$$
\widehat{C}_{x x}^{n}(t, x)=\mathbb{E}_{t, x}\left[\nabla h^{n}\left(\widetilde{S}_{1}^{n}\right)\left(\int_{t}^{1} \pi_{u}^{n} d W_{u}\right)\right], \quad(t, x) \in[0,1] \times(0, \infty),
$$

where $\pi^{n}$ is defined by

$$
\pi_{u}^{n}:=\frac{\nabla \widetilde{S}_{u}^{n}}{(1-t) \widehat{\gamma}^{n}\left(\widetilde{S}_{u}^{n}\right)}, \quad 0 \leq t \leq u \leq 1 .
$$

Proof. Fix any initial condition $(t, x) \in[0,1] \times(0, \infty)$ and $n \in \mathbb{N}$. Differentiating (4.13) with respect to $x$, we directly compute

$$
\widehat{C}_{x x}^{n}(t, x)=\mathbb{E}_{t, x}\left[\nabla^{2} h^{n}\left(\widetilde{S}_{1}^{n}\right) \nabla \widetilde{S}_{1}^{n}\right]=\mathbb{E}_{t, x}\left[\frac{1}{1-t} \int_{t}^{1} \nabla^{2} h^{n}\left(\widetilde{S}_{1}^{n}\right) D_{s} \widetilde{S}_{1}^{n} \frac{\nabla \widetilde{S}_{s}^{n}}{\hat{\gamma}\left(\widetilde{S}_{s}^{n}\right)} d s\right] .
$$


Recall that the Malliavin derivative and the tangent process only differ by their initial conditions. Hence, recalling the definition (4.17) of $\pi^{n}$, the integration by parts formula yields

$$
\widehat{C}_{x x}^{n}(t, x)=\mathbb{E}_{t, x}\left[\int_{t}^{1} D_{s}\left[\nabla h^{n}\left(\widetilde{S}_{1}^{n}\right)\right] \pi_{s}^{n} d s\right]=\mathbb{E}_{t, x}\left[\nabla h^{n}\left(\widetilde{S}_{1}^{n}\right) \int_{t}^{1} \pi_{s}^{n} d W_{s}\right] .
$$

Similarly, the third derivative of the price function also has such type of representation in expectation, where we emphasize that the stochastic integrals considered below are of Skorokhod type, since the integrand is not necessarily $\mathcal{F}$-adapted.

Lemma 4.3 For any $n \in \mathbb{N}$, we have

$$
\widehat{C}_{x x x}^{n}(t, x)=\mathbb{E}_{t, x}\left[\nabla h^{n}\left(\widetilde{S}_{1}^{n}\right)\left(\int_{t}^{1} \bar{\pi}_{u}^{n} d W_{u}\right)\right], \quad(t, x) \in[0,1] \times(0, \infty),
$$

where $\bar{\pi}^{n}$ is defined by

$$
\bar{\pi}_{u}^{n}:=\nabla_{x} \pi_{u}^{n}+\pi_{u}^{n}\left(\int_{t}^{1} \pi_{s}^{n} d W_{s}\right), \quad 0 \leq t \leq u \leq 1 .
$$

Proof. Fix any initial condition $(t, x) \in[0,1] \times(0, \infty)$ and $n \in \mathbb{N}$. Differentiating (4.16) with respect to $x$ and following a similar reasoning as above yields

$$
\begin{aligned}
\widehat{C}_{x x x}^{n}(t, x) & =\mathbb{E}_{t, x}\left[\nabla h^{n}\left(\widetilde{S}_{1}^{n}\right)\left(\int_{t}^{1} \nabla_{x} \pi_{s}^{n} d W_{s}\right)+\nabla^{2} h^{n}\left(\widetilde{S}_{1}^{n}\right) \nabla \widetilde{S}_{1}^{n}\left(\int_{t}^{1} \pi_{u}^{n} d W_{u}\right)\right] \\
& =\mathbb{E}_{t, x}\left[\nabla h\left(\widetilde{S}_{1}^{n}\right)\left(\int_{t}^{1} \nabla_{x} \pi_{s}^{n} d W_{s}\right)+\int_{t}^{1} D_{s}\left[\nabla h^{n}\left(\widetilde{S}_{1}^{n}\right)\right] \pi_{s}^{n}\left(\int_{t}^{1} \pi_{u}^{n} d W_{u}\right) d s\right] .
\end{aligned}
$$

Hence, the Malliavin integration by parts formula provides

$\widehat{C}_{x x x}^{n}(t, x)=\mathbb{E}_{t, x}\left[\nabla h\left(\widetilde{S}_{1}^{n}\right)\left(\int_{t}^{1} \nabla_{x} \pi_{s}^{n} d W_{s}\right)+\nabla h^{n}\left(\widetilde{S}_{1}^{n}\right) \int_{t}^{1} \pi_{s}^{n}\left(\int_{t}^{1} \pi_{u}^{n} d W_{u}\right) d W_{s}\right]$, and the definition (4.19) concludes the proof.

The exact same line of arguments provides a similar representation for the fourth derivative of the pricing function.

Lemma 4.4 For any $n \in \mathbb{N}$, we have

$$
\widehat{C}_{x x x x}^{n}(t, x)=\mathbb{E}_{t, x}\left[\nabla h^{n}\left(\widetilde{S}_{1}^{n}\right)\left(\int_{t}^{1} \hat{\pi}_{u}^{n} d W_{u}\right)\right], \quad(t, x) \in[0,1] \times(0, \infty),
$$

where $\hat{\pi}^{n}$ is defined by

$$
\hat{\pi}_{u}^{n}:=\nabla_{x} \bar{\pi}_{u}^{n}+\pi_{u}^{n}\left(\int_{t}^{1} \bar{\pi}_{s}^{n} d W_{s}\right), \quad 0 \leq t \leq u \leq 1 .
$$

These representations allow to derive estimates on the dependance of the derivatives of the pricing function $\hat{C}^{n}$, in terms of the parameter $n$. The rather computational obtention of these estimates is reported in Section 5 below. 
Proposition 4.5 There exist a constant $C$ and a continuous function $f$ on $(0, \infty)$ which do not depend on $n \in \mathbb{N}$, such that

$$
\begin{aligned}
\left|\widehat{C}_{x}^{n}(t, x)\right| & \leq C, \\
0 \leq \widehat{C}_{x x}^{n}(t, x) & \leq \frac{C}{\sqrt{(1-t) \gamma_{n}}} x^{-1 / 2} \\
\left|\widehat{C}_{x x x}^{n}(t, x)\right| & \leq \frac{C}{\gamma_{n}(1-t)} x^{-1}+\frac{C}{\sqrt{\gamma_{n}(1-t)}} x^{-3 / 2}, \\
\left|\widehat{C}_{x x x x}^{n}(t, x)\right| & \leq \frac{f(x)}{\sqrt{1-t} \gamma_{n}}+\frac{f(x)}{(1-t) \gamma_{n}}+\frac{f(x)}{(1-t)^{5 / 4} \gamma_{n}^{5 / 4}}+\frac{f(x)}{(1-t)^{3 / 2} \gamma_{n}^{3 / 2}}, \\
\left|\widehat{C}_{x t}^{n}(t, x)\right| & \leq \frac{f(x)}{(1-t)^{4 / 3} \ln (n)},
\end{aligned}
$$

for any $(t, x) \in[0,1] \times(0, \infty)$ and $n \in \mathbb{N}$.

Remark 4.7 Observe that (4.23) also indicates that the price function $\widehat{C}^{n}$ is convex with respect to the space variable. Indeed, the pricing function inherits the convexity of the payoff. This observation is crucial in order to ensure that a volatility upgrade allows to compensate the transaction costs.

\subsection{Asymptotics of the hedging error}

The subsection is dedicated to the proof of Theorem 3.4, the main result of the paper. We verify below that the sequence $\left(V_{1}^{n}\right)_{n}$ of terminal values for approximate replicating portfolios converges to $h\left(S_{1}\right)$, as the number of trading dates $n$ tends to infinity.

For any $n \in \mathbb{N}$, we rewrite the hedging strategy $\left(H_{t}^{n}\right)_{0 \leq t \leq 1}$ as $H^{n}=\hat{H}^{n}+K^{n}$ with

$$
\hat{H}_{t}^{n}:=\widehat{C}_{x}^{n}\left(t_{i}^{n}, S_{t_{i}^{n}}\right) \text { and } K_{t}^{n}:=\sum_{j \leq i} \widehat{C}_{x}^{n}\left(t_{j-1}^{n}, S_{t_{j-1}^{n}}\right)-\widehat{C}_{x}^{n}\left(t_{j}^{n}, S_{t_{j-1}^{n}}\right),
$$

for $t \in\left[t_{i}^{n}, t_{i+1}^{n}\right)$ and $1 \leq i \leq n$. We also denote $\Delta \hat{H}_{t}^{n}:=\hat{H}_{t+}^{n}-\hat{H}_{t-}^{n}$ and $\Delta K_{t}^{n}:=$ $K_{t+}^{n}-K_{t-}^{n}$. Therefore the terminal value of the candidate replicating portfolio $V_{1}^{n}$ rewrites

$$
V_{1}^{n}=\widehat{C}^{n}\left(0, S_{0}\right)+\int_{0}^{1} H_{u}^{n} d S_{u}-\sum_{i<n} G\left(t_{i}^{n}, \Delta \hat{H}_{t_{i}^{n}}^{n}+\Delta K_{t_{i}^{n}}^{n}\right), \quad n \in \mathbb{N} .
$$

Besides, the dynamics of $\widehat{C}^{n}$ and the definition (3.3) of $\hat{\sigma}^{n}$ yields

$$
h^{n}\left(S_{1}\right)=\widehat{C}^{n}\left(0, S_{0}\right)+\int_{0}^{1} \widehat{C}_{x}^{n}\left(u, S_{u}\right) d S_{u}+\frac{1}{2} \int_{0}^{1} \sigma \gamma_{n} S_{u} \widehat{C}_{x x}^{n}\left(u, S_{u}\right) d u, \quad n \in \mathbb{N} .
$$

Plugging the two expressions above together directly leads to the following tractable decomposition of the hedging error

$$
V_{1}^{n}-h\left(S_{1}\right)=F_{0}^{n}+F_{1}^{n}+F_{2}^{n}+F_{3}^{n}+F_{4}^{n},
$$


for any $n \in \mathbb{N}$, where

$$
\begin{aligned}
& F_{0}^{n}:=h^{n}\left(S_{1}\right)-h\left(S_{1}\right)+\int_{t_{n-1}^{n}}^{1}\left(\hat{H}_{t}^{n}-\widehat{C}_{x}^{n}\left(t, S_{t}\right)\right) d S_{t}-G\left(1, \Delta \hat{H}_{1}^{n}+\Delta K_{1}^{n}\right), \\
& F_{1}^{n}:=\int_{0}^{t_{n-1}^{n}}\left(\hat{H}_{t}^{n}-\widehat{C}_{x}^{n}\left(t, S_{t}\right)\right) d S_{t}, \\
& F_{2}^{n}:=\int_{0}^{1} K_{t}^{n} d S_{t} \\
& F_{3}^{n}:=\sum_{i=1}^{n-1} G^{\prime}(0)\left|\Delta \hat{H}_{t_{i}^{n}}^{n}+\Delta K_{t_{i}^{n}}^{n}\right|-\sum_{i=1}^{n-1} G\left(t_{i}^{n}, \Delta \hat{H}_{t_{i}^{n}}^{n}+\Delta K_{t_{i}^{n}}^{n}\right), \\
& F_{4}^{n}:=\frac{1}{2} \int_{0}^{1} \sigma \gamma_{n} S_{t} \widehat{C}_{x x}^{n}\left(t, S_{t}\right) d t-\sum_{i=1}^{n-1} G^{\prime}(0)\left|\Delta \hat{H}_{t_{i}^{n}}^{n}+\Delta K_{t_{i}^{n}}^{n}\right| .
\end{aligned}
$$

We now prove that each sequence of random variables $\left(F_{j}^{n}\right)_{n}$ for $j=0, \ldots, 4$ goes to zero in probability, as $n$ goes to infinity.

Proposition 4.6 The sequences $\left(F_{0}^{n}\right),\left(F_{1}^{n}\right),\left(F_{2}^{n}\right)$ and $\left(F_{3}^{n}\right)$ converge to 0 in probability as $n$ goes to $\infty$.

Proof. We prove the convergence of each sequence separately.

\section{Step 0. Convergence of $\left(F_{0}^{n}\right)_{n}$.}

By construction of $\left(h_{n}\right),(3.7)$ implies that the first term $h^{n}\left(S_{1}\right)-h\left(S_{1}\right)$ tends to 0 as $h^{n} \rightarrow h$. The second one converges to 0 because $\left(\widehat{C}_{x}^{n}(., S .)\right)_{n}$ is bounded according to (4.22). As for the last term, observe from (4.13) that

$$
\begin{aligned}
\left|\Delta \hat{H}_{1}^{n}\right| & =\left|\widehat{C}_{x}^{n}\left(1, S_{1}\right)-\widehat{C}_{x}^{n}\left(t_{n-1}^{n}, S_{t_{n-1}^{n}}\right)\right|=\left|\nabla h^{n}\left(S_{1}\right)-\mathbb{E}\left[\nabla h^{n}\left(\tilde{S}_{1}^{n}\right) \mid \tilde{S}_{t_{n-1}^{n}}^{n}=S_{t_{n-1}^{n}}\right]\right| \\
& \leq\left\|\nabla^{2} h^{n}\right\|_{\infty} \mathbb{E}\left[\left|S_{1}-\tilde{S}_{1}^{n}\right| \mid \tilde{S}_{t_{n-1}^{n}}^{n}=S_{t_{n-1}^{n}}\right], \quad n \in \mathbb{N} .
\end{aligned}
$$

As $\mathbb{E}\left|S_{1}-S_{t_{n-1}^{n}}\right| \leq C \sqrt{1 / n}$, we deduce from (3.7) that

$$
\left|\Delta \hat{H}_{1}^{n}\right| \leq C \frac{\gamma_{n}^{1 / 6}}{\sqrt{n} \ln n}+C \frac{\gamma_{n}^{1 / 6}}{\ln n} \mathbb{E}\left|\tilde{S}_{1}^{n}-\tilde{S}_{t_{n-1}^{n}}^{n}\right|, \quad n \in \mathbb{N} .
$$

From the dynamics (4.12) of $\tilde{S}^{n}$, we compute directly $\mathbb{E}\left|\tilde{S}_{1}^{n}-\tilde{S}_{t_{n-1}^{n}}^{n}\right| \leq C \sqrt{\gamma_{n} / n}$ so that $\left|\Delta \hat{H}_{1}^{n}\right|$ goes to 0 as $n$ goes to infinity. Very similarly, we show that $\left|\Delta K_{1}^{n}\right|$ converges also to 0 and Condition $(\mathbf{G})$ provides the convergence of $F_{0}^{n}$ to 0 .

Step 1. Convergence of $\left(F_{1}^{n}\right)_{n}$.

Applying the Ito formula, we directly compute that

$$
\hat{H}_{t}^{n}-\widehat{C}_{x}^{n}\left(t, S_{t}\right)=M_{t}^{n}-M_{t_{i}^{n}}^{n}+A_{t}^{n}-A_{t_{i}^{n}}^{n}, \quad t_{i}^{n} \leq t<t_{i+1}^{n}, \quad i<n-1,
$$

where the sequence of processes $\left(M^{n}\right)_{n}$ and $\left(A^{n}\right)_{n}$ are given by

$M^{n}:=\int_{0} \sigma S_{u} \widehat{C}_{x x}^{n}\left(u, S_{u}\right) d W_{u}$ and $A^{n}:=\int_{0}^{\cdot}\left[\widehat{C}_{x t}^{n}\left(u, S_{u}\right)+\frac{1}{2} \sigma^{2} S_{u}^{2} \widehat{C}_{x x x}^{n}\left(u, S_{u}\right)\right] d u$, 
for any $n \in \mathbb{N}$. Since $S$ has bounded moments, (4.23) together with the CauchySchwartz inequality yield

$$
\mathbb{E}\left(M_{t}^{n}-M_{t_{i}^{n}}^{n}\right)^{4} \leq \frac{C}{\gamma_{n}^{2}}\left(\int_{t_{i}^{n}}^{t_{i+1}^{n}} \frac{d u}{1-u}\right)^{2} \leq \frac{C}{\gamma_{n}^{2}}, \quad t_{i}^{n} \leq t<t_{i+1}^{n}, \quad i<n-1 .
$$

Besides, (4.24) together with (4.26) indicate that

$$
\begin{aligned}
\mathbb{E}\left|A_{t}^{n}-A_{t_{i}^{n}}^{n}\right|^{4} & \leq C\left(\int_{t_{i}^{n}}^{t} \frac{\ln (n)^{-1} d u}{(1-u)^{4 / 3}}+\frac{\gamma_{n}^{-1} d u}{(1-u)}+\frac{\gamma_{n}^{-1 / 2} d u}{\sqrt{1-u}}\right)^{4} \\
& \leq C\left(\frac{\ln (n)^{-1}}{n(1-t)^{4 / 3}}+\frac{\gamma_{n}^{-1 / 2}}{n(1-t)}\right)^{4}, \quad t_{i}^{n} \leq t<t_{i+1}^{n}, \quad i<n-1 .
\end{aligned}
$$

Plugging the last two estimates in (4.29) leads directly to

$$
\mathbb{E}\left|F_{1}^{n}\right|^{2} \leq \frac{C}{\gamma_{n}}+\frac{C}{n^{2}} \int_{0}^{t_{n-1}^{n}}\left(\frac{|\ln (n)|^{-2}}{(1-t)^{8 / 3}}+\frac{\gamma_{n}^{-1}}{(1-t)^{2}}\right) d t \leq \frac{C}{\gamma_{n}}+\frac{C|\ln (n)|^{-2}}{n^{1 / 3}}+\frac{C}{n \gamma_{n}},
$$

for any $n \in \mathbb{N}$, so that $\mathbb{E}\left|F_{1}^{n}\right|^{2}$ goes to 0 as $n$ goes to infinity.

Step 2. Convergence of $\left(F_{2}^{n}\right)_{n}$.

From the definition of $K^{n}$ given in (4.27), we directly compute

$$
F_{2}^{n}=-\sum_{i \leq n-1} \int_{t_{i-1}^{n}}^{t_{i}^{n}} \widehat{C}_{x t}^{n}\left(u, S_{t_{i-1}^{n}}\right)\left(S_{t_{i}^{n}}-S_{1}\right) d u
$$

Combining the Cauchy-Schwartz inequality together with (4.26) yields

$$
\begin{aligned}
\mathbb{E}\left|F_{2}^{n}\right| & \leq \frac{C}{\ln n} \sum_{i \leq n-1} \mathbb{E}\left[\left|S_{t_{i}^{n}}-S_{1}\right|^{2}\right]^{1 / 2} \int_{t_{i-1}^{n}}^{t_{i}^{n}} \frac{d u}{(1-u)^{4 / 3}} \\
& \leq \frac{C}{n \ln n} \sum_{i \leq n-1} \frac{1}{\left(1-t_{i}^{n}\right)^{5 / 6}} \leq \frac{C}{\ln n} \rightarrow 0 .
\end{aligned}
$$

Step 3. Convergence of $\left(F_{3}^{n}\right)_{n}$.

For any $0 \leq i \leq n$, observe that

$$
\Delta \hat{H}_{t_{i}^{n}}^{n}+\Delta K_{t_{i}^{n}}^{n}=\widehat{C}_{x}^{n}\left(t_{i}^{n}, S_{t_{i}^{n}}\right)-\widehat{C}_{x}^{n}\left(t_{i}^{n}, S_{t_{i-1}^{n}}\right)=\widehat{C}_{x x}^{n}\left(t_{i}^{n}, \bar{S}_{t_{i}^{n}}\right)\left(S_{t_{i}^{n}}-S_{t_{i-1}^{n}}\right),
$$

where the random variable $\bar{S}_{t_{i}^{n}}$ is between $S_{t_{i-1}^{n}}$ and $S_{t_{i}^{n}}$. Hence, (4.23) together with Condition (G) yield $\left|F_{3}^{n}\right|^{i} \leq C_{\omega} \chi_{3}^{n}$ where

$$
\chi_{3}^{n}:=\sum_{i \leq n-1} \frac{1}{\gamma_{n}\left(1-t_{i}^{n}\right)}\left(S_{t_{i}^{n}}-S_{t_{i-1}^{n}}\right)^{2},
$$

for any $n \in \mathbb{N}$. But $\mathbb{E} \chi_{3}^{n} \leq C\left(\gamma_{n}\right)^{-1} \ln n$, hence $F_{3}^{n} \rightarrow 0$ as $n$ goes to $\infty$.

Proposition 4.7 The sequence $\left(F_{4}^{n}\right)$ converges to 0 in probability as $n$ goes to $\infty$. 
Proof. For any $n \in \mathbb{N}$, we write $F_{4}^{n}=\sum_{i=1}^{4} L_{i}^{n}$ with the summands

$$
\begin{aligned}
& L_{1}^{n}:=\frac{1}{2} \int_{0}^{1} \sigma \gamma_{n} S_{t} \widehat{C}_{x x}^{n}\left(t, S_{t}\right) d t-\frac{1}{2 n} \sum_{i=1}^{n-1} \sigma \gamma_{n} S_{t_{i-1}^{n}} \widehat{C}_{x x}^{n}\left(t_{i-1}^{n}, S_{t_{i-1}^{n}}\right), \\
& L_{2}^{n}:=\sum_{i=1}^{n-1} \widehat{C}_{x x}^{n}\left(t_{i-1}^{n}, S_{t_{i-1}^{n}}\right)\left(\frac{\sigma \gamma_{n}}{2 n}-G^{\prime}(0) \sigma\left|\Delta W_{t_{i}^{n}}\right|\right), \\
& L_{3}^{n}:=\sigma G^{\prime}(0) \sum_{i=1}^{n-1}\left(S_{t_{i-1}^{n}} \widehat{C}_{x x}^{n}\left(t_{i-1}^{n}, S_{t_{i-1}^{n}}\right)\left|\Delta W_{t_{i}^{n}}\right|-\left|\int_{t_{i-1}^{n}}^{t_{i}^{n}} \sigma S_{u} \widehat{C}_{x x}^{n}\left(u, S_{u}\right) d W_{u}\right|\right), \\
& L_{4}^{n}:=G^{\prime}(0) \sum_{i=1}^{n-1}\left|\int_{t_{i-1}^{n}}^{t_{i}^{n}} \sigma S_{u} \widehat{C}_{x x}^{n}\left(u, S_{u}\right) d W_{u}\right|-G^{\prime}(0) \sum_{i=1}^{n-1}\left|\Delta H_{t_{i}^{n}}^{n}+\Delta K_{t_{i}^{n}}^{n}\right| .
\end{aligned}
$$

Observe that the previous decomposition uses the convexity of the price function given in (4.23), see Remark 4.7.

It now suffices to show that $L_{i}^{n} \rightarrow 0$ for $i=1, \ldots 4$ as detailed in the steps below.

Step 1. Convergence of $\left(L_{1}^{n}\right)_{n}$.

We have $\left|L_{1}^{n}\right| \leq C_{\omega}\left(\left|L_{11}^{n}\right|+\left|L_{12}^{n}\right|\right)$ where, by virtue of (4.23),

$$
\begin{aligned}
L_{11}^{n} & :=\gamma_{n} \sum_{i=1}^{n-1} \int_{t_{i-1}^{n}}^{t_{i}^{n}} \frac{\left(S_{t}-S_{t_{i-1}^{n}}\right)}{\sqrt{\gamma_{n}(1-t)}} d t, \\
L_{12}^{n} & :=\gamma_{n} \sum_{i=1}^{n-1} \int_{t_{i-1}^{n}}^{t_{i}^{n}} S_{t_{i-1}^{n}}\left(\widehat{C}_{x x}^{n}\left(t, S_{t}\right)-\widehat{C}_{x x}^{n}\left(t_{i-1}^{n}, S_{t_{i-1}^{n}}\right)\right) d t .
\end{aligned}
$$

We have $\mathbb{E}\left|L_{11}^{n}\right| \leq C \sqrt{\gamma_{n} / n} \rightarrow 0$. For the second term, we use the Taylor expansion $\widehat{C}_{x x}^{n}\left(t, S_{t}\right)-\widehat{C}_{x x}^{n}\left(t_{i-1}^{n}, S_{t_{i-1}^{n}}\right)=\widehat{C}_{x x x}\left(\bar{t}_{i}^{n}, \bar{S}_{t_{i}^{n}}\right)\left(S_{t}-S_{t_{i-1}^{n}}\right)+\widehat{C}_{x x t}\left(\bar{t}_{i}^{n}, \bar{S}_{t_{i}^{n}}\right)\left(t-t_{i-1}^{n}\right)$, for some random variables $\bar{t}_{i}^{n}$ and $\bar{S}_{t_{i}^{n}}$, for $t_{i-1}^{n} \leq t<t_{i}^{n}$. Besides, differentiating the dynamics of $\widehat{C}^{n}$, we observe that

$$
\widehat{C}_{x t t}^{n}=-2 \sigma^{2} \widehat{C}_{x x}^{n}-\left(2 \sigma^{2} x+\sigma \gamma_{n} x\right) \widehat{C}_{x x x}^{n}-\left(\sigma^{2} x^{2}+\sigma \gamma_{n} x\right) \widehat{C}_{x x x x}^{n},
$$

for any $x \in(0, \infty)$ and $n \in \mathbb{N}$. Hence, combining (4.23), (4.24) and (4.25), we get

$$
\begin{aligned}
L_{12}^{n} & \leq C_{\omega} \gamma_{n} \sum_{i=1}^{n-1} \int_{t_{i-1}^{n}}^{t_{i}^{n}} \frac{S_{t}-S_{t_{i-1}^{n}}}{\gamma_{n}(1-t)} d t \\
& +C_{\omega} \frac{\gamma_{n}^{2}}{n} \int_{0}^{t_{n-1}^{n}}\left(\frac{d t}{\sqrt{\gamma_{n}(1-t)}}+\frac{d t}{\gamma_{n}(1-t)}+\frac{d t}{\gamma_{n}^{5 / 4}(1-t)^{5 / 4}}+\frac{d t}{\gamma_{n}^{3 / 2}(1-t)^{3 / 2}}\right),
\end{aligned}
$$

for any $n \in \mathbb{N}$. Hence the Cauchy-Schwartz inequality and a direct computation yield

$$
\mathbb{E} L_{12}^{n} \leq C\left(\frac{\ln (n)}{\sqrt{n}}+\frac{1}{n^{1 / 4}}+\frac{1}{n^{3 / 8}}\right) \rightarrow 0 .
$$

Step 2. Convergence of $\left(L_{2}^{n}\right)_{n}$. 
We use the equality $E\left|\Delta W_{t_{i}^{n}}\right|=\sqrt{2 / \pi n}$ from which we deduce

$$
\mathbb{E}\left(\frac{\sigma \gamma_{n}}{2 n}-G^{\prime}(0) \sigma\left|\Delta W_{t_{i}^{n}}\right|\right)^{2}=\operatorname{Var}\left[G^{\prime}(0) \sigma\left|\Delta W_{t_{i}^{n}}\right|\right]=\frac{\sigma^{2} G^{\prime}(0)^{2}}{n},
$$

for any $i \leq n$. The independence of the increments of the Brownian motion together with (4.23) yield

$$
\mathbb{E}\left(L_{2}^{n}\right)^{2} \leq \frac{C}{n} \sum_{i \leq n-1} \frac{1}{\gamma_{n}\left(1-t_{i}^{n}\right)} \leq \frac{C \ln (n)}{n \sqrt{n}} \rightarrow 0 .
$$

Step 3. Convergence of $\left(L_{3}^{n}\right)_{n}$.

We use the inequality ||$a|-| b|| \leq|a-b|$. Therefore, the Cauchy-Schwarz inequality and the Ito isometry give us

$$
\mathbb{E}\left|L_{3}^{n}\right| \leq C \sum_{i=1}^{n-1}\left(\int_{t_{i-1}^{n}}^{t_{i}^{n}} \mathbb{E}\left[\left|S_{t_{i-1}^{n}} \widehat{C}_{x x}^{n}\left(t_{i-1}^{n}, S_{t_{i-1}^{n}}\right)-S_{u} \widehat{C}_{x x}^{n}\left(u, S_{u}\right)\right|^{2}\right] d u\right)^{1 / 2}
$$

By the Ito formula, we get $d\left[S_{t} \widehat{C}_{x x}^{n}\left(t, S_{t}\right)\right]=f_{t}^{n} d W_{t}+g_{t}^{n} d t$ where

$$
\begin{aligned}
f_{t}^{n} & :=\sigma S_{t} \widehat{C}_{x x}^{n}\left(t, S_{t}\right)+\sigma S_{t}^{2} \widehat{C}_{x x x}^{n}\left(t, S_{t}\right), \\
g_{t}^{n} & :=S_{t} \widehat{C}_{x x t}^{n}\left(t, S_{t}\right)+\frac{1}{2} \sigma^{2} S_{t}^{3} \widehat{C}_{x x x x}^{n}\left(t, S_{t}\right)+\sigma^{2} S_{t}^{2} \widehat{C}_{x x x}^{n}\left(t, S_{t}\right),
\end{aligned}
$$

for $0 \leq t \leq 1$ and $n \in \mathbb{N}$. Hence, we derive

$$
\mathbb{E}\left|L_{3}^{n}\right| \leq C \sum_{i=1}^{n-1}\left(\frac{1}{n} \int_{t_{i-1}^{n}}^{t_{i}^{n}} \mathbb{E}\left|f_{s}^{n}\right|^{2} d s+\frac{2}{n^{2}} \int_{t_{i-1}^{n}}^{t_{i}^{n}} \mathbb{E}\left|g_{s}^{n}\right|^{2} d s\right)^{1 / 2} .
$$

Estimates (4.23) and (4.24) provide

$$
\int_{t_{i-1}^{n}}^{t_{i}^{n}} \mathbb{E}\left|f_{u}^{n}\right|^{2} d u \leq \frac{C}{n}\left(\frac{1}{\sqrt{\left(1-t_{i}^{n}\right) \gamma_{n}}}+\frac{1}{\left(1-t_{i}^{n}\right) \gamma_{n}}\right)^{2}, \quad i \leq n .
$$

Besides, combining (4.30) together with (4.23), (4.24) and (4.25), we get

$$
\int_{t_{i-1}^{n}}^{t_{i}^{n}} \mathbb{E}\left|g_{u}^{n}\right|^{2} d u \leq \frac{C \gamma_{n}}{n}\left(\frac{\gamma_{n}^{-1 / 2}}{\sqrt{\left(1-t_{i}^{n}\right)}}+\frac{\gamma_{n}^{-1}}{\left(1-t_{i}^{n}\right)}+\frac{\gamma_{n}^{-5 / 4}}{\left(1-t_{i}^{n}\right)^{5 / 4}}+\frac{\gamma_{n}^{-3 / 2}}{\left(1-t_{i}^{n}\right)^{3 / 2}}\right) .
$$

Plugging these last two estimates in (4.31), similar computations as in Step 1 yield to the convergence of $\mathbb{E}\left|L_{3}^{n}\right|$ to zero.

\section{Step 4. Convergence of $\left(L_{4}^{n}\right)_{n}$.}

We first verify that we may replace $\Delta K^{n}$ by $\Delta \tilde{K}^{n}$ where

$$
\Delta \tilde{K}_{t_{i}^{n}}^{n}:=-\int_{t_{i-1}^{n}}^{t_{i}^{n}} \widehat{C}_{x t}^{n}\left(u, S_{u}\right) d u, \quad i \leq n .
$$

To do so, it suffices to show that $\chi^{n} \rightarrow 0$ where

$$
\chi^{n}:=\sum_{i \leq n-1} \int_{t_{i-1}^{n}}^{t_{i}^{n}}\left(\widehat{C}_{x t}^{n}\left(u, S_{u}\right)-\widehat{C}_{x t}^{n}\left(u, S_{t_{i-1}^{n}}\right)\right) d u .
$$


Using a Taylor expansion, we compute

$$
\widehat{C}_{x t}^{n}\left(u, S_{u}\right)-\widehat{C}_{x t}^{n}\left(u, S_{t_{i-1}^{n}}\right)=\widehat{C}_{x x t}^{n}\left(u, \bar{S}_{t_{i-1}^{n}}\right)\left(S_{u}-S_{t_{i-1}^{n}}\right),
$$

for some random variable $\bar{S}_{t_{i-1}^{n}}$ between $S_{u}$ and $S_{t_{i-1}^{n}}$, for any $0 \leq t_{i}^{n} \leq u \leq 1$. Hence (4.30) together with (4.23), (4.24) and (4.25) imply that $\chi^{n} \leq C_{\omega} \bar{\chi}^{n}$ where

$\bar{\chi}^{n}:=\gamma_{n} \sum_{i=1}^{n-1} \int_{t_{i-1}^{n}}^{t_{i}^{n}}\left(\frac{S_{u}-S_{t_{i-1}^{n}}}{\sqrt{1-t} \gamma_{n}^{1 / 2}}+\frac{S_{u}-S_{t_{i-1}^{n}}}{(1-t) \gamma_{n}}+\frac{S_{u}-S_{t_{i-1}^{n}}}{(1-t)^{5 / 4} \gamma_{n}^{5 / 4}}+\frac{S_{u}-S_{t_{i-1}^{n}}}{(1-t)^{3 / 2} \gamma_{n}^{3 / 2}}\right) d u$,

for $n \in \mathbb{N}$. As $\mathbb{E}\left|S_{u}-S_{t_{i-1}^{n}}\right| \leq C n^{-1 / 2}$ for $t_{i}^{n} \leq u \leq t_{i+1}^{n}$, we easily conclude that $\mathbb{E} \bar{\chi}^{n} \rightarrow 0$. At last, replacing $\Delta K_{t_{i}^{n}}^{n}$ by $\Delta \tilde{K}_{t_{i}^{n}}^{n}$ and using the inequality ||$a|-| b|| \leq$ $|a-b|$, we deduce from Ito's formula together with (4.24) that

$$
\left|L_{4}^{n}\right| \leq C_{\omega} \int_{0}^{t_{n-1}^{n}} \widehat{C}_{x x x}\left(u, S_{u}\right) d u \leq c_{\omega} \int_{0}^{t_{n-1}^{n}}\left(\frac{d u}{\sqrt{(1-u) \gamma_{n}}}+\frac{d u}{(1-u) \gamma_{n}}\right) \rightarrow 0 .
$$

\section{Price sensitivities estimation}

This section is dedicated to the obtention of the estimates presented in Proposition 4.5 above, which allow to upper bound the sensitivities of the price function $\widehat{C}^{n}$ in terms of the number of trading dates $n$. The control of each sensitivity is presented separately. These estimates, namely (4.22), (4.23), (4.24), (4.25) and (4.26), are obtained using the Malliavin representation of the Greeks detailed in Section 4.1. This particular feature is new in the classical scheme of proof for the obtention of Leland type convergence theorems.

In all the section, we fix $(t, x) \in[0,1] \times(0, \infty)$ and omit the subscript $\{t, x\}$ in order to alleviate the notations.

5.1 Estimates (4.22) and (4.23) on the first and second derivatives

First observe that estimate (4.22) directly follows from the representation (4.13), since $\left(\nabla h^{n}\right)_{n}$ is bounded. The rest of this subsection is dedicated to the obtention of $(4.23)$.

We fix $(t, x) \in[0,1] \times(0, \infty)$. Using (4.16) together with the Cauchy-Schwartz inequality, we derive

$$
\left|\hat{C}_{x x}^{n}(t, x)\right| \leq\left\|\nabla h^{n}\right\|_{\infty}\left(\int_{t}^{1} \mathbb{E}\left|\pi_{u}^{n}\right|^{2} d u\right)^{1 / 2}, \quad n \in \mathbb{N} .
$$

We now focus more closely on the dynamics of the processes $\left(\pi^{n}\right)_{n}$ defined by (4.17). First, according to the dynamics of $\widetilde{S}^{n}$, the tangent process $\nabla \widetilde{S}^{n}$ satisfies

$$
d \nabla \widetilde{S}_{u}^{n}=\nabla \widehat{\gamma}_{n}\left(\widetilde{S}_{u}^{n}\right) \nabla \widetilde{S}_{u}^{n} d W_{u}+\left(\left|\nabla \widehat{\gamma}_{n}\left(\widetilde{S}_{u}^{n}\right)\right|^{2}+\widehat{\gamma}_{n}\left(\widetilde{S}_{u}^{n}\right) \nabla^{2} \widehat{\gamma}_{n}\left(\widetilde{S}_{u}^{n}\right)\right) \nabla \widetilde{S}_{u}^{n} d u,
$$


for $n \in \mathbb{N}$. Besides, Ito's formula implies that $1 / \widehat{\gamma}_{n}\left(\widetilde{S}^{n}\right)$ has the following dynamics

$$
\begin{aligned}
d\left(\frac{1}{\widehat{\gamma}_{n}\left(\widetilde{S}_{u}^{n}\right)}\right) & =-\frac{\nabla \widehat{\gamma}_{n}\left(\widetilde{S}_{u}^{n}\right)}{\left|\widehat{\gamma}_{n}\left(\widetilde{S}_{u}^{n}\right)\right|^{2}} d \widetilde{S}_{u}^{n}+\frac{2\left|\nabla \widehat{\gamma}_{n}\left(\widetilde{S}_{u}^{n}\right)\right|^{2}-\widehat{\gamma}_{n}\left(\widetilde{S}_{u}^{n}\right) \nabla^{2} \widehat{\gamma}_{n}\left(\widetilde{S}_{u}^{n}\right)}{2 \widehat{\gamma}_{n}\left(\widetilde{S}_{u}^{n}\right)} d u \\
& =-\frac{\nabla \widehat{\gamma}_{n}\left(\widetilde{S}_{u}^{n}\right)}{\left|\widehat{\gamma}_{n}\left(\widetilde{S}_{u}^{n}\right)\right|^{2}} d W_{u}-\frac{\nabla^{2} \widehat{\gamma}_{n}\left(\widetilde{S}_{u}^{n}\right)}{2} d u, \quad n \in \mathbb{N} .
\end{aligned}
$$

A direct application of the integration by parts formula hence implies

$$
d \pi_{u}^{n}=\frac{\nabla^{2} \widehat{\gamma}_{n}\left(\widetilde{S}_{u}^{n}\right) \nabla \widetilde{S}_{u}^{n}}{2(1-t)} d u=-\frac{\sigma^{2} \gamma_{n}^{2}}{8\left|\widehat{\gamma}_{n}\left(\widetilde{S}_{u}^{n}\right)\right|^{2}} \pi_{u}^{n} d u, \quad n \in \mathbb{N} .
$$

Therefore, we deduce that

$$
\pi_{u}^{n}=\pi_{t}^{n} \exp \left\{-\int_{t}^{u} \frac{\sigma^{2} \gamma_{n}^{2}}{8\left|\widehat{\gamma}_{n}\left(\widetilde{S}_{s}^{n}\right)\right|^{2}} d s\right\} \leq \frac{1}{(1-t) \widehat{\gamma}_{n}(x)}, \quad 0 \leq u \leq 1, \quad n \in \mathbb{N} .
$$

Plugging this expression together with $\widehat{\gamma}_{n}(x) \geq \sqrt{\sigma \gamma_{n} x}$ in (5.32) provides (4.23). Indeed (5.34) also indicates that $\pi^{n}$ and hence $\nabla \tilde{S}^{n}$ are non-negative, so that $\widehat{C}_{x x}^{n}(t, x)=\mathbb{E}_{t, x}\left[\nabla^{2} h^{n}\left(\widetilde{S}_{1}^{n}\right) \nabla \widetilde{S}_{1}^{n}\right] \geq 0$.

\subsection{Estimate (4.24) on the third derivative}

This subsection is dedicated to the obtention of (4.24) and divides in 3 steps.

\section{Step 1. Estimate decomposition}

Using (4.18), we derive

$$
\left|\widehat{C}_{x x x}^{n}(t, x)\right| \leq\left\|\nabla h^{n}\right\|_{\infty} \mathbb{E}\left|\bar{Z}_{1}^{n}\right| \quad \text { where } \bar{Z}_{1}^{n}:=\int_{t}^{1} \bar{\pi}_{u}^{n} d W_{u}, \quad n \in \mathbb{N} .
$$

Let us introduce the sequence of processes $\left(Z^{n}\right)_{n}$ given by

$$
Z_{s}^{n}:=\int_{t}^{s} \pi_{u}^{n} d W_{u}, \quad n \in \mathbb{N} .
$$

By the definition of $\left(\bar{\pi}^{n}\right)$ given in (4.19), we compute

$$
\begin{aligned}
\bar{Z}_{1}^{n} & =\int_{t}^{1} \nabla_{x} \pi_{u}^{n} d W_{u}+\int_{t}^{1} \pi_{u}^{n} Z_{1}^{n} d W_{u} \\
& =\int_{t}^{1} \nabla_{x} \pi_{u}^{n} d W_{u}+\left|Z_{1}^{n}\right|^{2}-\int_{t}^{1} \pi_{u}^{n} D_{u} Z_{1}^{n} d u \\
& =\int_{t}^{1} \nabla_{x} \pi_{u}^{n} d W_{u}+\left|Z_{1}^{n}\right|^{2}-\int_{t}^{1}\left|\pi_{u}^{n}\right|^{2} d u-\int_{t}^{1} \pi_{u}^{n}\left(\int_{u}^{1} D_{u} \pi_{s}^{n} d W_{s}\right) d u \\
& =\left|Z_{1}^{n}\right|^{2}-\int_{t}^{1}\left|\pi_{u}^{n}\right|^{2} d u+\int_{t}^{1}\left(\nabla_{x} \pi_{u}^{n}-\int_{t}^{u} \pi_{s}^{n} D_{s} \pi_{u}^{n} d s\right) d W_{u}, \quad n \in \mathbb{N} .
\end{aligned}
$$


Plugging this expression in (5.35) and using Ito's formula, we deduce

$$
\left|\widehat{C}_{x x x}^{n}(t, x)\right| \leq C\left(A_{n}^{1 / 2}+B_{n}\right), \quad n \in \mathbb{N},
$$

where $\left(A_{n}\right)$ and $\left(B_{n}\right)$ are respectively defined by

$$
A_{n}:=\mathbb{E}\left|\int_{t}^{1} \pi_{u}^{n} Z_{u}^{n} d W_{u}\right|^{2} \quad \text { and } \quad B_{n}:=\mathbb{E}\left|\int_{t}^{1}\left(\nabla_{x} \pi_{u}^{n}-\int_{t}^{u} \pi_{s}^{n} D_{s} \pi_{u}^{n} d s\right) d W_{u}\right|,
$$

for $n \in \mathbb{N}$. We now fix $n \in \mathbb{N}$ and intend to control the terms $A_{n}$ and $B_{n}$ separately.

Step 2. Control of $\left(A_{n}\right)_{n}$

Recall from (5.34) that $\left|\pi^{n}\right| \leq 1 /(1-t) \widehat{\gamma}_{n}(x)$. Hence, we get from a direct application of Ito's formula that

$$
A_{n}=\mathbb{E}\left|\int_{t}^{1} \pi_{u}^{n}\left(\int_{t}^{u} \pi_{s}^{n} d W_{s}\right) d W_{u}\right|^{2}=\int_{t}^{1} \mathbb{E}\left|\pi_{u}^{n}\left(\int_{t}^{u} \pi_{s}^{n} d W_{s}\right)\right|^{2} d u
$$

We recall from (5.34) that $\left|\pi^{n}\right| \leq 1 /(1-t) \widehat{\gamma}_{n}(x)$ and deduce from the previous expression

$$
A_{n}=\int_{t}^{1} \mathbb{E}\left|\pi_{u}^{n}\left(\int_{t}^{u} \pi_{s}^{n} d W_{s}\right)\right|^{2} d u \leq \frac{1}{(1-t)^{2}\left|\widehat{\gamma}_{n}(x)\right|^{2}} \int_{t}^{1} \int_{t}^{u} \mathbb{E}\left|\pi_{s}^{n}\right|^{2} d s d u .
$$

Using once again the same relation together with $\left|\hat{\gamma}_{n}(x)\right|^{2} \geq \sigma \gamma_{n} x$ yields

$$
A_{n}^{1 / 2} \leq \frac{1}{2(1-t)^{2}\left|\widehat{\gamma}_{n}(x)\right|^{4}} \leq \frac{1}{\sqrt{2} \sigma(1-t) \gamma_{n} x}
$$

Step 3. Control of $\left(B_{n}\right)_{n}$

We now turn to the more intricate term $B_{n}$. Let us introduce the notation

$$
b^{n}:=\nabla_{x} \pi^{n}-\int_{t}\left[D_{s} \pi_{u}^{n}\right] \pi_{s}^{n} d s, \quad \text { so that } \quad B_{n}=\mathbb{E}\left|\int_{t}^{1} b_{u}^{n} d W_{u}\right| .
$$

By virtue of the martingale moment inequalities, there exists $C>0$ such that

$$
B_{n} \leq C \mathbb{E}\left(\int_{t}^{1}\left|b_{u}^{n}\right|^{2} d u\right)^{1 / 2} \leq C \sqrt{1-t} \mathbb{E} \sup _{t \leq u \leq 1}\left|b_{u}^{n}\right|, \quad n \in \mathbb{N}
$$

In order to control the last term on the r. h. s. , we look towards the dynamics of $\left(b^{n}\right)_{n}$. Differentiating the dynamics of $\left(\pi^{n}\right)$ given in (5.33), we compute separately

$$
\begin{aligned}
& d \nabla_{x} \pi_{u}^{n}=-\frac{\sigma^{2} \gamma_{n}^{2}}{8\left|\widehat{\gamma}_{n}\left(\widetilde{S}_{u}^{n}\right)\right|^{2}} \nabla_{x} \pi_{u}^{n} d u+\frac{\sigma^{2} \gamma_{n}^{2} \nabla \widehat{\gamma}_{n}\left(\widetilde{S}_{u}^{n}\right)}{4\left|\widehat{\gamma}_{n}\left(\widetilde{S}_{u}^{n}\right)\right|^{3}} \nabla \widetilde{S}_{u}^{n} \pi_{u}^{n} d u, \\
& d D_{s} \pi_{u}^{n}=-\frac{\sigma^{2} \gamma_{n}^{2}}{8\left|\widehat{\gamma}_{n}\left(\widetilde{S}_{u}^{n}\right)\right|^{2}} D_{s} \pi_{u}^{n} d u+\frac{\sigma^{2} \gamma_{n}^{2} \nabla \widehat{\gamma}_{n}\left(\widetilde{S}_{u}^{n}\right)}{4\left|\widehat{\gamma}_{n}\left(\widetilde{S}_{u}^{n}\right)\right|^{3}} D_{s} \widetilde{S}_{u}^{n} \pi_{u}^{n} d u, \quad t \leq s,
\end{aligned}
$$


Since $D_{s} \widetilde{S}_{r}^{n}=\nabla \widetilde{S}_{r}^{n} \widehat{\gamma}_{n}\left(\widetilde{S}_{s}^{n}\right) / \nabla \widetilde{S}_{s}^{n}=\nabla \widetilde{S}_{r}^{n} /\left\{(1-t) \pi_{s}^{n}\right\}$ for $t \leq s \leq r \leq 1$, we deduce

$$
\begin{aligned}
\int_{t}^{u} \pi_{s}^{n} D_{s} \pi_{u}^{n} d s & =-\int_{t}^{u} \int_{s}^{u} \frac{\sigma^{2} \gamma_{n}^{2} \pi_{s}^{n}}{8\left|\widehat{\gamma}_{n}\left(\widetilde{S}_{r}^{n}\right)\right|^{2}} D_{s} \pi_{r}^{n} d r d s+\int_{t}^{u} \int_{s}^{u} \frac{\sigma^{2} \gamma_{n}^{2} \nabla \widehat{\gamma}_{n}\left(\widetilde{S}_{r}^{n}\right)}{4\left|\widehat{\gamma}_{n}\left(\widetilde{S}_{r}^{n}\right)\right|^{3}} D_{s} \widetilde{S}_{r}^{n} \pi_{r}^{n} \pi_{s}^{n} d r d s \\
& =-\int_{t}^{u}\left(\int_{t}^{r} \pi_{s}^{n} D_{s} \pi_{r}^{n} d s\right) \frac{\sigma^{2} \gamma_{n}^{2} d r}{8\left|\widehat{\gamma}_{n}\left(\widetilde{S}_{r}^{n}\right)\right|^{2}}+\int_{t}^{u} \frac{r-t}{1-t} \frac{\sigma^{2} \gamma_{n}^{2} \nabla \widehat{\gamma}_{n}\left(\widetilde{S}_{r}^{n}\right)}{4\left|\widehat{\gamma}_{n}\left(\widetilde{S}_{r}^{n}\right)\right|^{3}} \nabla \widetilde{S}_{r}^{n} \pi_{r}^{n} d r,
\end{aligned}
$$

for $t \leq u \leq 1$. Combining this expression with (5.42), we get

$$
d b_{u}^{n}=-\frac{\sigma^{2} \gamma_{n}^{2}}{8\left|\widehat{\gamma}_{n}\left(\widetilde{S}_{u}^{n}\right)\right|^{2}} b_{u}^{n} d u+\frac{\sigma^{2} \gamma_{n}^{2} \nabla \widehat{\gamma}_{n}\left(\widetilde{S}_{u}^{n}\right)}{4\left|\widehat{\gamma}_{n}\left(\widetilde{S}_{u}^{n}\right)\right|^{2}}\left|\pi_{u}^{n}\right|^{2}(1-u) d u .
$$

Notice that $b_{t}^{n}=-\nabla \widehat{\gamma}_{n}(x) /(1-t)\left|\widehat{\gamma}_{n}(x)\right|^{2}<0$. From the dynamics of $b^{n}$, we observe that $b^{n}$ increases as long as $b^{n}$ is negative. Once it becomes positive, it must remain non negative, since the negative part of the drift disappears as soon as $b^{n}$ reaches 0 . Indeed, $b^{n}=L^{n} \pi^{n} / \pi_{t}^{n}$ where

$$
L^{n}:=b_{t}^{n}+\int_{t}^{\cdot} \frac{\sigma^{2} \gamma_{n}^{2} \nabla \widehat{\gamma}_{n}\left(\widetilde{S}_{r}^{n}\right)}{4\left|\widehat{\gamma}_{n}\left(\widetilde{S}_{r}^{n}\right)\right|^{2}} \pi_{r}^{n} \pi_{t}^{n}(1-r) d r
$$

is strictly increasing. From there, we deduce that $b^{n}$ and $L^{n}$ have the same sign. Hence $b^{n}$ is always non negative on $\left[\tau^{n}, 1\right]$ where $\tau^{n}:=\inf \left\{s \in[t, 1], \quad b_{s}^{n}=0\right\} \wedge 1$. Therefore, we get

$$
\begin{aligned}
\left|b_{u}^{n}\right| & \leq-b_{t}^{n} \mathbf{1}_{\left\{b_{u} \leq 0\right\}}+b_{u}^{n} \mathbf{1}_{\left\{u \geq \tau^{n}\right\}} \\
& \leq-b_{t}^{n}-\mathbf{1}_{\left\{u \geq \tau^{n}\right\}} \int_{\tau^{n}}^{u} \frac{\sigma^{2} \gamma_{n}^{2} b_{r}^{n}}{8\left|\widehat{\gamma}_{n}\left(\widetilde{S}_{r}^{n}\right)\right|^{2}} d r+\mathbf{1}_{\left\{u \geq \tau^{n}\right\}} \int_{\tau^{n}}^{u} \frac{\sigma^{2} \gamma_{n}^{2} \nabla \widehat{\gamma}_{n}\left(\widetilde{S}_{r}^{n}\right)}{4\left|\widehat{\gamma}_{n}\left(\widetilde{S}_{r}^{n}\right)\right|^{2}}\left|\pi_{r}^{n}\right|^{2}(1-r) d r,
\end{aligned}
$$

for any $t \leq u \leq 1$, which directly leads to

$$
\left|b_{u}^{n}\right| \leq\left|b_{t}^{n}\right|+\Gamma_{u}^{n}, \quad \text { with } \quad \Gamma^{n}:=-\int_{t}^{\cdot} \nabla \widehat{\gamma}_{n}\left(\widetilde{S}_{r}^{n}\right)(1-r) 2 \pi_{r}^{n} d \pi_{r}^{n} .
$$

Since $\nabla \widehat{\gamma}_{n}$ is non-negative and $\pi^{n}$ is decreasing, we deduce that

$$
\mathbb{E} \sup _{t \leq u \leq 1}\left|b_{u}^{n}\right| \leq \frac{\nabla \widehat{\gamma}_{n}(x)}{(1-t)\left|\widehat{\gamma}_{n}(x)\right|^{2}}+\mathbb{E} \Gamma_{1}^{n} .
$$

We now focus on the last term of this expression and observe from a direct application of the integration by parts formula that

$$
\begin{aligned}
\Gamma_{u}^{n}= & (1-t) \nabla \widehat{\gamma}_{n}(x)\left|\pi_{t}^{n}\right|^{2}-(1-u) \nabla \widehat{\gamma}_{n}\left(\widetilde{S}_{u}^{n}\right)\left|\pi_{u}^{n}\right|^{2} \\
& +\int_{t}^{u}\left|\pi_{r}^{n}\right|^{2}(1-r) d \nabla \widehat{\gamma}_{n}\left(\widetilde{S}_{r}^{n}\right)-\int_{t}^{u}\left|\pi_{r}^{n}\right|^{2} \nabla \widehat{\gamma}_{n}\left(\widetilde{S}_{r}^{n}\right) d r, \quad t \leq u \leq 1 .
\end{aligned}
$$

We compute

$$
\nabla \widehat{\gamma}_{n}(x)=\frac{2 \sigma^{2} x+\sigma \gamma_{n}}{2 \sqrt{\sigma^{2} x^{2}+\sigma \gamma_{n} x}}, \nabla^{2} \widehat{\gamma}_{n}(x)=-\frac{\sigma^{2} \gamma_{n}^{2}}{4 \widehat{\gamma}_{n}^{3}(x)}, \nabla^{3} \widehat{\gamma}_{n}(x)=\frac{3 \sigma^{2} \gamma_{n}^{2}}{4} \frac{\nabla \widehat{\gamma}_{n}(x)}{\widehat{\gamma}_{n}^{4}(x)},
$$


and deduce from the application of Ito's formula that

$$
d \nabla \widehat{\gamma}_{n}\left(\widetilde{S}_{u}^{n}\right)=\frac{-\sigma^{2} \gamma_{n}^{2}}{4 \widehat{\gamma}_{n}^{2}\left(\widetilde{S}_{u}^{n}\right)}\left(d W_{u}-\frac{\nabla \widehat{\gamma}_{n}\left(\widetilde{S}_{u}^{n}\right)}{2} d u\right)=\frac{-\sigma^{2} \gamma_{n}^{2}}{4 \widehat{\gamma}_{n}^{2}\left(\widetilde{S}_{u}^{n}\right)} d W_{u}-\nabla \gamma\left(\widetilde{S}_{u}^{n}\right) \frac{d \pi_{u}^{n}}{\pi_{u}^{n}}
$$

Plugging this expression in (5.48) directly leads to

$$
\Gamma_{u}^{n} \leq(1-t) \nabla \widehat{\gamma}_{n}(x)\left|\pi_{t}^{n}\right|^{2}+N_{u}^{n}+\frac{1}{2} \Gamma_{u}^{n}, \quad t \leq u \leq 1
$$

where $N^{n}:=-\int_{t}\left|\pi_{r}^{n}\right|^{2}(1-r) \frac{\sigma^{2} \gamma_{n}^{2}}{4 \widehat{\gamma}_{n}^{2}\left(\widetilde{S}_{r}^{n}\right)} d W_{r}$. Since $\Gamma^{n} \geq 0$, it follows that $\left(N_{u}^{n}\right)_{u \geq t}$ is a supermartingale whence $E N_{1}^{n} \leq 0$. We deduce an upper bound on $E \Gamma_{1}^{n}$ which plugged in (5.47) provides

$$
\mathbb{E} \sup _{t \leq u \leq 1}\left|b_{u}^{n}\right| \leq \frac{\nabla \widehat{\gamma}_{n}(x)}{(1-t)\left|\widehat{\gamma}_{n}(x)\right|^{2}}+2(1-t) \nabla \widehat{\gamma}_{n}(x)\left|\pi_{t}^{n}\right|^{2}=\frac{3 \nabla \widehat{\gamma}_{n}(x)}{(1-t)\left|\widehat{\gamma}_{n}(x)\right|^{2}}
$$

Together with (5.41) and the expression $\nabla \widehat{\gamma}_{n}(x) / \widehat{\gamma}_{n}(x) \leq C / x$, we get

$$
B_{n} \leq \frac{C}{x \widehat{\gamma}_{n}(x) \sqrt{1-t}} \leq \frac{C}{\sqrt{\gamma_{n}(1-t)}} x^{-3 / 2}
$$

which, combined with (5.37) and (5.39), provides (4.24).

5.3 Estimate (4.25) on the fourth derivative

This subsection is dedicated to the obtention of (4.25). Fix $n \in \mathbb{N}$. The representation (4.20) directly provides

$$
\left|\hat{C}_{x x x x}(t, x)\right| \leq\left\|\nabla h^{n}\right\|_{\infty} \mathbb{E}\left|\int_{t}^{1} \hat{\pi}_{u}^{n} d W_{u}\right|
$$

and we now intend to control the term $\mathbb{E}\left|\int_{t}^{1} \hat{\pi}_{u}^{n} d W_{u}\right|$ in several steps.

Step 1. A tractable Decomposition for $\mathbb{E}\left|\int_{t}^{1} \hat{\pi}_{u}^{n} d W_{u}\right|$.

Let introduce the notation

$$
\bar{Z}_{u}^{n}:=\int_{t}^{u}\left(b_{s}^{n}+2 \pi_{s}^{n} Z_{s}^{n}\right) d W_{s}, \quad t \leq u \leq 1, \text { so that } \bar{Z}_{1}^{n}=\int_{t}^{1} \bar{\pi}_{s}^{n} d W_{s},
$$

where $\left(b^{n}\right)_{n}$ is defined above and given by $b^{n}:=\nabla \pi^{n}-\int_{t}^{\cdot} \pi_{r}^{n}\left(D_{r} \pi^{n}\right) d r$. The definition of $\hat{\pi}^{n}$ given in (4.21) implies

$$
\int_{t}^{1} \hat{\pi}_{u}^{n} d W_{u}=\int_{t}^{1} \nabla \bar{\pi}_{u}^{n} d W_{u}+\int_{t}^{1} \pi_{u}^{n} \bar{Z}_{1}^{n} d W_{u}=\nabla \bar{Z}_{1}^{n}+\int_{t}^{1} \pi_{u}^{n} \bar{Z}_{1}^{n} d W_{u} .
$$


Using integration by parts formulae, observe that $\int_{t}^{1} \pi_{u}^{n} \bar{Z}_{1}^{n} d W_{u}$ rewrites

$$
\begin{aligned}
& Z_{1}^{n} \bar{Z}_{1}^{n}-\int_{t}^{1} \pi_{u}^{n} D_{u}\left[\bar{Z}_{1}^{n}\right] d u \\
= & Z_{1}^{n} \bar{Z}_{1}^{n}-\int_{t}^{1} \pi_{u}^{n}\left(b_{u}^{n}+2 \pi_{u}^{n} Z_{u}^{n}\right) d u-\int_{t}^{1} \pi_{u}^{n}\left(\int_{u}^{1}\left(D_{u} b_{s}^{n}+2 D_{u}\left[\pi_{s}^{n} Z_{s}^{n}\right]\right) d W_{s}\right) d u \\
= & \int_{t}^{1} Z_{u}^{n} d \bar{Z}_{u}^{n}+\int_{t}^{1} \bar{Z}_{u}^{n} d Z_{u}^{n}-\int_{t}^{1}\left(\int_{t}^{s} \pi_{u}^{n} D_{u} b_{s}^{n} d u-2 Z_{s}^{n} \int_{t}^{s} \pi_{u}^{n} D_{u} \pi_{s}^{n} d u\right) d W_{s} \\
& -2 \int_{t}^{1} \pi_{s}^{n}\left(\int_{t}^{s}\left|\pi_{u}^{n}\right|^{2} d u\right) d W_{s}-2 \int_{t}^{1} \pi_{s}^{n}\left(\int_{t}^{s}\left(\int_{t}^{r} \pi_{u}^{n} D_{u} \pi_{r}^{n} d u\right) d W_{r}\right) d W_{s} .
\end{aligned}
$$

Plugging this expression together with $\nabla \bar{Z}_{1}^{n}=\int_{t}^{1}\left(\nabla b_{s}^{n}+2 Z_{s}^{n} \nabla \pi_{s}^{n}+2 \pi_{s}^{n} \nabla Z_{s}^{n}\right) d W_{s}$ and the definition of $b^{n}$ in (5.52), we obtain

$$
\begin{aligned}
\int_{t}^{1} \hat{\pi}_{u}^{n} d W_{u} & =\int_{t}^{1} c_{s}^{n} d W_{s}+\int_{t}^{1} Z_{u}^{n} d \bar{Z}_{u}^{n}+\int_{t}^{1} \bar{Z}_{u}^{n} d Z_{u}^{n} \\
& +2 \int_{t}^{1}\left\{Z_{s}^{n} b_{s}^{n}+\pi_{s}^{n}\left(\int_{t}^{s} b_{r}^{n} d W_{r}\right)-\pi_{s}^{n}\left(\int_{t}^{s}\left|\pi_{u}^{n}\right|^{2} d u\right)\right\} d W_{s}
\end{aligned}
$$

where $c^{n}:=\nabla b^{n}-\int_{t} \pi_{r}^{n}\left(D_{r} b^{n}\right) d r$. Introducing the dynamics of $Z^{n}$ and $\bar{Z}^{n}$ in the previous expression, we get

$$
\begin{aligned}
\int_{t}^{1} \hat{\pi}_{u}^{n} d W_{u} & =\int_{t}^{1} c_{s}^{n} d W_{s}+3 \int_{t}^{1}\left(Z_{s}^{n} b_{s}^{n}+\pi_{s}^{n} \int_{t}^{s} b_{r}^{n} d W_{r}\right) d W_{s} \\
& +2 \int_{t}^{1} \pi_{s}^{n}\left(\left|Z_{s}^{n}\right|^{2}+\int_{t}^{s} \pi_{r}^{n} Z_{r}^{n} d W_{r}-\int_{t}^{s}\left|\pi_{r}^{n}\right|^{2} d r\right) d W_{s} .
\end{aligned}
$$

Using Ito's formula together with the definition of $\bar{Z}^{n}$, we deduce

$$
\mathbb{E}\left|\int_{t}^{1} \hat{\pi}_{u}^{n} d W_{u}\right| \leq 3 C_{1}^{n}+3 C_{2}^{n}+C_{3}^{n}
$$

where we set

$C_{1}^{n}:=\mathbb{E}\left|\int_{t}^{1} \pi_{s}^{n}\left(\int_{t}^{s} \bar{Z}_{r}^{n} d W_{r}\right) d W_{s}\right|, C_{2}^{n}:=\mathbb{E}\left|\int_{t}^{1} Z_{s}^{n} b_{s}^{n} d W_{s}\right|, C_{3}^{n}:=\mathbb{E}\left|\int_{t}^{1} c_{s}^{n} d W_{s}\right|$.

We now require to control these three terms separately.

\section{Step 2. Control of $\left(C_{1}^{n}\right)$}

Using twice the martingale moment inequality, we compute

$$
\begin{aligned}
C_{1}^{n} & \leq C \pi_{t}^{n} \mathbb{E} \sqrt{\int_{t}^{1}\left(\bar{Z}_{u}^{n}\right)^{2} d u} \leq c \pi_{t}^{n} \sqrt{1-t} \mathbb{E} \sup _{t \leq u \leq 1}\left|\bar{Z}_{u}^{n}\right| \\
& \leq C \pi_{t}^{n} \sqrt{1-t}\left(\sqrt{1-t} \mathbb{E} \sup _{t \leq u \leq t}\left|b_{u}^{n}\right|+2 \pi_{t}^{n} \sqrt{1-t} \mathbb{E} \sup _{t \leq u \leq 1}\left|Z_{u}^{n}\right|\right) \\
& \leq C \pi_{t}^{n}(1-t)\left(\mathbb{E} \sup _{t \leq u \leq t}\left|b_{u}^{n}\right|+2 c\left|\pi_{t}^{n}\right|^{2} \sqrt{1-t}\right) .
\end{aligned}
$$


Plugging (5.50) in this expression, it follows that

$$
C_{1}^{n} \leq \frac{C}{\widehat{\gamma}_{n}(x)(1-t)}\left(\frac{\nabla \widehat{\gamma}_{n}(x)}{\left|\widehat{\gamma}_{n}(x)\right|^{2}}+\frac{1}{\sqrt{1-t}\left|\widehat{\gamma}_{n}(x)\right|^{2}}\right) .
$$

Since $\left|\widehat{\gamma}_{n}(x)\right|^{2} \geq \sigma \gamma_{n} x$ and $\left|\nabla \widehat{\gamma}_{n}(x)\right| /\left|\widehat{\gamma}_{n}(x)\right| \leq 3 / 2 x$, we deduce that

$$
C_{1}^{n} \leq \frac{C}{(1-t) \gamma_{n} x}\left(\frac{1}{x}+\frac{1}{\sqrt{(1-t) \gamma_{n} x}}\right) .
$$

Step 3. Control of $\left(C_{2}^{n}\right)$

Applying the martingale moment inequality together with the relation (5.46), we deduce

$$
C_{2}^{n} \leq C \sqrt{1-t} \mathbb{E} \sup _{t \leq u \leq 1}\left|b_{u}^{n} Z_{u}^{n}\right| \leq C \sqrt{1-t}\left(\left|b_{t}^{n}\right| \mathbb{E} \sup _{t \leq u \leq 1}\left|Z_{u}^{n}\right|+\mathbb{E} \sup _{t \leq u \leq 1} \Gamma_{u}^{n}\left|Z_{u}^{n}\right|\right),
$$

where $\Gamma^{n}$ defined in (5.46) is non negative and increasing. Using once again the martingale moment inequality, we derive

$$
C_{2}^{n} \leq C(1-t)\left|b_{t}^{n}\right| \pi_{t}^{n}+C \sqrt{1-t} \mathbb{E} \sup _{t \leq u \leq 1} \Gamma_{u}^{n}\left|Z_{u}^{n}\right|
$$

Observe that the integration by parts formula yields

$$
d \Gamma_{u}^{n} Z_{u}^{n}=-\nabla \widehat{\gamma}_{n}\left(\widetilde{S}_{u}^{n}\right)(1-u) Z_{u}^{n} 2 \pi_{u}^{n} d \pi_{u}^{n}+\Gamma_{u}^{n} \pi_{u}^{n} d W_{u}
$$

The Jensen inequality applied to the concave function $x \mapsto \sqrt{x}$ yields the inequality $\left(\int f(u) u d u\right)^{2} \leq\left(\int f(u) d u\right)\left(\int f(u) u^{2} d u\right)$. Since $\pi^{n}$ is decreasing, we deduce that

$$
2 \sup _{t \leq u \leq 1}\left|\Gamma_{u}^{n} Z_{u}^{n}\right| \leq \sup _{t \leq u \leq 1}\left|\int_{t}^{u} \Gamma_{r}^{n} \pi_{r} d W_{r}\right|+\left|\Gamma_{1}^{n} \bar{\Gamma}_{1}^{n}\right|^{1 / 2}
$$

where, using (5.49), we have

$$
\begin{aligned}
\bar{\Gamma}_{u}^{n}:= & -\int_{t}^{u} \nabla \widehat{\gamma}_{n}\left(\widetilde{S}_{r}^{n}\right)(1-r)\left|Z_{r}^{n}\right|^{2} 2 \pi_{r}^{n} d \pi_{r}^{n} \\
= & \int_{t}^{u}\left|\pi_{r}^{n}\right|^{4} \nabla \widehat{\gamma}_{n}\left(\widetilde{S}_{r}^{n}\right)(1-r) d r-\int_{t}^{u}\left|\pi_{r}^{n}\right|^{2}\left|Z_{r}^{n}\right|^{2} \nabla \widehat{\gamma}_{n}\left(\widetilde{S}_{r}^{n}\right) d r \\
& -\int_{t}^{u}\left|\pi_{r}^{n}\right|^{2}\left|Z_{r}^{n}\right|^{2}(1-r) \nabla \widehat{\gamma}_{n}\left(\widetilde{S}_{r}^{n}\right) \frac{d \pi_{r}^{n}}{\pi_{r}^{n}}+2 \int_{t}^{u}\left(\pi_{r}^{n}\right)^{3}(1-r) d\left\langle\left|Z^{n}\right|^{2}, \nabla \widehat{\gamma}_{n}\left(\widetilde{S}^{n}\right)\right\rangle_{r}+N_{u}^{n},
\end{aligned}
$$

for $t \leq u \leq 1$, with $N^{n}$ a local martingale. Hence, we deduce that $\frac{1}{2} \bar{\Gamma}_{u}^{n} \leq N_{u}^{n}+$ $\chi_{1}^{n}+\chi_{2}^{n}$ where

$$
\chi_{1}^{n}:=\int_{t}^{1}\left(\pi_{r}^{n}\right)^{4} \nabla \widehat{\gamma}_{n}\left(\widetilde{S}_{r}^{n}\right)(1-r) d r \geq 0, \quad \chi_{2}^{n}:=-4 \int_{t}^{1}(1-r)\left|Z_{r}^{n}\right|\left|\pi_{r}^{n}\right|^{2} d \pi_{r}^{n} \geq 0 .
$$

Applying Ito's formula to $\left(\left|\pi_{r}^{n}\right|^{4} \nabla \widehat{\gamma}_{n}\left(\widetilde{S}_{r}^{n}\right)(1-r)^{2}\right)_{t \leq r \leq 1}$ together with the relation (5.49) yields

$$
\begin{aligned}
\chi_{1}^{n}= & \left|\pi_{t}^{n}\right|^{4} \nabla \widehat{\gamma}_{n}(x)(1-t)^{2}+4 \int_{t}^{1}(1-r)^{2} \nabla \widehat{\gamma}_{n}\left(\widetilde{S}_{r}^{n}\right)\left(\pi_{r}^{n}\right)^{3} d \pi_{r}^{n} \\
& -\int_{t}^{1}(1-r)^{2}\left(\pi_{r}^{n}\right)^{4} \nabla \widehat{\gamma}_{n}\left(\widetilde{S}_{r}^{n}\right) \frac{d \pi_{r}^{n}}{\pi_{r}^{n}}+N_{1}^{1, n},
\end{aligned}
$$


where $N^{1, n}$ is a lower bounded local martingale, so that

$$
\mathbb{E}\left|\chi_{1}^{n}\right| \leq\left|\pi_{t}^{n}\right|^{4} \nabla \widehat{\gamma}_{n}(x)(1-t)^{2} .
$$

From the martingale inequality together with Ito's formula, we get

$$
\mathbb{E}\left|\chi_{2}^{n}\right| \leq 4 \mathbb{E} \sup _{r}\left|Z_{r}^{n}\right| \int_{t}^{1}-(1-r)\left|\pi_{r}^{n}\right|^{2} d \pi_{r}^{n} \leq 4 \sqrt{1-t} \pi_{t}^{n}(1-t) \frac{\left|\pi_{t}^{n}\right|^{3}}{3}
$$

where the last inequality follows from the monotonicity of $\pi^{n}$ together with Doob's inequality.

We deduce that $\mathbb{E}\left|\chi_{1}^{n}+\chi_{2}^{n}\right|<\infty$, so that $0 \leq \frac{1}{2} \bar{\Gamma}_{u}^{n} \leq N_{u}^{n}+\mathbb{E}\left[\left|\chi_{1}^{n}+\chi_{2}^{n}\right| \mathcal{F}_{u}\right]$, which implies that $N^{n}$ is a supermartingale. Therefore $\mathbb{E} N_{1}^{n} \leq 0$ and $\mathbb{E} \bar{\Gamma}_{1}^{n} \leq 2 \mathbb{E}\left[\chi_{1}^{n}+\chi_{2}^{n}\right]$. Hence, the two previous inequalities together with (5.56) lead to

$$
2 \mathbb{E} \sup _{u}\left|\Gamma_{u}^{n} Z_{u}^{n}\right| \leq \mathbb{E} \sup _{u}\left|\int_{t}^{u} \Gamma_{r}^{n} \pi_{r}^{n} d W_{r}\right|+\sqrt{\mathbb{E} \Gamma_{1}^{n}} \sqrt{\frac{\left|\pi_{t}^{n}\right|^{4}}{(1-t)^{-2}} \nabla \widehat{\gamma}_{n}(x)+\frac{4}{3} \frac{\left(\pi_{t}^{n}\right)^{4}}{(1-t)^{-3 / 2}}} .
$$

The martingale moment inequality and the monotonicity of $\Gamma^{n}$ and $\pi^{n}$ ensure

$$
\mathbb{E} \sup _{t \leq u \leq 1}\left|\int_{t}^{u} \Gamma_{r}^{n} \pi_{r}^{n} d W_{r}\right| \leq C \mathbb{E} \sqrt{\int_{t}^{u}\left|\Gamma_{r}^{n} \pi_{r}^{n}\right|^{2} d r} \leq C \pi_{t}^{n} \sqrt{1-t} \mathbb{E} \Gamma_{1}^{n} .
$$

Plugging $\mathbb{E} \Gamma_{1}^{n} \leq 2(1-t) \nabla \widehat{\gamma}_{n}(x)\left|\pi_{t}^{n}\right|^{2}$ observed in (5.50) together with the definitions of $\pi_{t}^{n}$ and $b_{t}^{n}$ in the previous expressions and (5.55) leads to

$$
C_{2}^{n} \leq \frac{C}{\sqrt{1-t}}\left(\frac{\nabla \widehat{\gamma}_{n}(x)}{\widehat{\gamma}_{n}(x)^{3} \sqrt{1-t}}+\frac{\nabla \widehat{\gamma}_{n}(x)}{\widehat{\gamma}_{n}(x)^{2}}+\frac{\nabla \widehat{\gamma}_{n}(x)}{\widehat{\gamma}_{n}(x)^{4}(1-t)}+\frac{1}{\widehat{\gamma}_{n}(x)^{4}(1-t)^{3 / 2}}\right)
$$

Since $\nabla \widehat{\gamma}_{n}(x) / \widehat{\gamma}_{n}(x) \leq 3 / 2 x$ and $\widehat{\gamma}_{n}(x)^{2} \geq \sigma \gamma_{n} x$, we compute

$$
C_{2}^{n} \leq \frac{f(x)}{(1-t) \gamma_{n}}+\frac{f(x)}{(1-t)^{5 / 4} \gamma_{n}^{5 / 4}}
$$

for some continuous function $f$.

\section{Step 4. Control of $C_{3}^{n}$}

We now turn to the last term $C_{3}^{n}$ and observe from the martingale moment inequality that

$$
C_{3}^{n}=\mathbb{E}\left|\int_{t}^{1} c_{s}^{n} d W_{s}\right| \leq C \mathbb{E} \sqrt{\int_{t}^{1}\left|c_{s}^{n}\right|^{2} d s} \leq C \sqrt{1-t} \mathbb{E} \sup _{t \leq s \leq 1}\left|c_{s}^{n}\right| .
$$

In order to control this last term, we compute the dynamics of $c^{n}$ defined as $\nabla b^{n}-\int_{t} \pi_{s}^{n} D_{s} b^{n} d s$. We deduce from the dynamics of $b^{n}$ given in (5.44) that

$$
\begin{aligned}
d \nabla b_{u}^{n} & =-\frac{\gamma_{n}^{2}}{8\left|\widehat{\gamma}_{n}\left(\widetilde{S}_{u}^{n}\right)\right|^{2}} \nabla b_{u}^{n} d u+\frac{\gamma_{n}^{2}}{4} \frac{\nabla \widehat{\gamma}_{n}}{\left|\widehat{\gamma}_{n}\right|^{3}}\left(\widetilde{S}_{u}^{n}\right) \nabla \widetilde{S}_{u}^{n} b_{u}^{n} d u \\
& +\frac{\gamma_{n}^{2}}{2} \frac{\nabla \widehat{\gamma}_{n}}{\left|\widehat{\gamma}_{n}\right|^{2}}\left(\widetilde{S}_{u}^{n}\right) \pi_{u}^{n} \nabla \pi_{u}^{n}(1-u) d u+\frac{\gamma_{n}^{2}}{4} \nabla\left[\frac{\nabla \widehat{\gamma}_{n}}{\left|\widehat{\gamma}_{n}\right|^{2}}\right]\left(\widetilde{S}_{u}^{n}\right) \nabla \widetilde{S}_{u}^{n}\left|\pi_{u}^{n}\right|^{2}(1-u) d u .
\end{aligned}
$$


Similarly, we compute

$$
\begin{aligned}
d D_{s} b_{u}^{n} & =-\frac{\gamma_{n}^{2}}{8\left|\widehat{\gamma}_{n}\left(\widetilde{S}_{u}^{n}\right)\right|^{2}} D_{s} b_{u}^{n} d u+\frac{\gamma_{n}^{2}}{4} \frac{\nabla \widehat{\gamma}_{n}}{\left|\widehat{\gamma}_{n}\right|^{3}}\left(\widetilde{S}_{u}^{n}\right) D_{s} \widetilde{S}_{u}^{n} b_{u}^{n} d u \\
& +\frac{\gamma_{n}^{2}}{2} \frac{\nabla \widehat{\gamma}_{n}}{\left|\widehat{\gamma}_{n}\right|^{2}}\left(\widetilde{S}_{u}^{n}\right) \pi_{u}^{n} D_{s} \pi_{u}^{n}(1-u) d u+\frac{\gamma_{n}^{2}}{4} \nabla\left[\frac{\nabla \widehat{\gamma}_{n}}{\left|\widehat{\gamma}_{n}\right|^{2}}\right]\left(\widetilde{S}_{u}^{n}\right) D_{s} \widetilde{S}_{u}^{n}\left|\pi_{u}^{n}\right|^{2}(1-u) d u,
\end{aligned}
$$

for $t \leq s \leq u \leq 1$. Since $D . \widetilde{S}_{u}^{n}=\nabla \widetilde{S}^{n} /\left\{(1-t) \pi_{s}^{n}\right\}$, we deduce following the same line of arguments as in Step 3 of the previous section that

$$
\begin{aligned}
d c_{u}^{n} & =-\frac{\gamma_{n}^{2}}{8\left|\widehat{\gamma}_{n}\left(\widetilde{S}_{u}^{n}\right)\right|^{2}} c_{u}^{n} d u+\frac{\gamma_{n}^{2}}{4} \frac{\nabla \widehat{\gamma}_{n}}{\left|\widehat{\gamma}_{n}\right|^{3}}\left(\widetilde{S}_{u}^{n}\right) b_{u}^{n}\left(\frac{1-u}{1-t}\right) \nabla \widetilde{S}_{u}^{n} d u \\
& +\frac{\gamma_{n}^{2}}{2} \frac{\nabla \widehat{\gamma}_{n}}{\left|\widehat{\gamma}_{n}\right|^{2}}\left(\widetilde{S}_{u}^{n}\right) \pi_{u}^{n}(1-u) b_{u}^{n} d u+\frac{\gamma_{n}^{2}}{4} \nabla\left[\frac{\nabla \widehat{\gamma}_{n}}{\left|\widehat{\gamma}_{n}\right|^{2}}\right]\left(\widetilde{S}_{u}^{n}\right) \nabla \widetilde{S}_{u}^{n}\left|\pi_{u}^{n}\right|^{2} \frac{(1-u)^{2}}{1-t} d u
\end{aligned}
$$

Therefore, Ito's formula together with the definition of $\pi^{n}$ leads to

$$
\begin{aligned}
\frac{c_{u}^{n}}{\pi_{u}^{n}} & =\frac{c_{t}^{n}}{\pi_{t}^{n}}+6 \int_{t}^{u} \nabla \widehat{\gamma}_{n}\left(\widetilde{S}_{r}^{n}\right)(1-r) \frac{b_{r}^{n}}{\pi_{r}^{n}} \frac{\gamma_{n}^{2}}{8} \frac{\pi_{r}^{n} d r}{\left|\widehat{\gamma}_{n}\left(\widetilde{S}_{r}^{n}\right)\right|^{2}} \\
& +\int_{t}^{u} \frac{\gamma_{n}^{2}}{4}\left[\frac{\nabla^{2} \widehat{\gamma}_{n}}{\widehat{\gamma}_{n}}-2 \frac{\left|\nabla \widehat{\gamma}_{n}\right|^{2}}{\left|\widehat{\gamma}_{n}\right|^{2}}\right]\left(\widetilde{S}_{r}^{n}\right)\left|\pi_{r}^{n}\right|^{2}(1-r)^{2} d r, \quad t \leq u \leq 1 .
\end{aligned}
$$

Since $\pi^{n}$ and $\nabla^{2} \widehat{\gamma}_{n}$ are decreasing, this relation combined with (5.58) implies

$$
\begin{gathered}
C_{3}^{n} \leq C \sqrt{1-t}\left(\left|c_{t}^{n}\right|+\mathbb{E} X_{1}^{n}+\mathbb{E} Y_{1}^{n}\right), \\
\text { with } \quad X^{n}:=\int_{t}^{\cdot}-\nabla \widehat{\gamma}_{n}\left(\widetilde{S}_{r}^{n}\right)(1-r)\left|\frac{b_{r}^{n}}{\pi_{r}^{n}}\right| 2 \pi_{r}^{n} d \pi_{r}^{n} \geq 0, \\
Y^{n}:=\int_{t}^{\cdot}-\left(2\left|\nabla \widehat{\gamma}_{n}\left(\widetilde{S}_{r}^{n}\right)\right|^{2}-\nabla^{2} \widehat{\gamma}_{n}\left(\widetilde{S}_{r}^{n}\right) \widehat{\gamma}_{n}\left(\widetilde{S}_{r}^{n}\right)\right)\left|\pi_{r}^{n}\right|^{2}(1-r)^{2} d \pi_{r}^{n} \geq 0 .
\end{gathered}
$$

We first focus on the process $Y^{n}$ and, since $\pi^{n}$ is decreasing, observe that

$$
0 \leq Y^{n} \leq\left|\pi_{t}^{n}\right|^{2} \int_{t}^{\cdot}-\left(2\left|\nabla \widehat{\gamma}_{n}\left(\widetilde{S}_{r}^{n}\right)\right|^{2}-\nabla^{2} \widehat{\gamma}_{n}\left(\widetilde{S}_{r}^{n}\right) \widehat{\gamma}_{n}\left(\widetilde{S}_{r}^{n}\right)\right)(1-r)^{2} d \pi_{r}^{n}
$$

Applying Ito's formula to the process $\left((1-u)^{2} \pi_{u}^{n} / \widehat{\gamma}_{n}\left(\widetilde{S}_{u}^{n}\right)^{2}\right)_{t \leq u \leq 1}$, we get

$$
\begin{gathered}
\frac{(1-u)^{2} \pi_{u}^{n}}{\left|\widehat{\gamma}_{n}\left(\widetilde{S}_{u}^{n}\right)\right|^{2}}-\frac{(1-t)^{2} \pi_{t}^{n}}{\left|\widehat{\gamma}_{n}(x)\right|^{2}}=\int_{t}^{u}\left(3 \frac{\left|\nabla \widehat{\gamma}_{n}\left(\widetilde{S}_{r}^{n}\right)\right|^{2}}{\widehat{\gamma}_{n}\left(\widetilde{S}_{r}^{n}\right)^{4}}-\frac{\nabla^{2} \widehat{\gamma}_{n}\left(\widetilde{S}_{r}^{n}\right)}{\widehat{\gamma}_{n}\left(\widetilde{S}_{r}^{n}\right)^{3}}\right) \frac{\pi_{r}^{n}\left|\widehat{\gamma}_{n}\left(\widetilde{S}_{r}^{n}\right)\right|^{2}}{(1-r)^{-2}} d r \\
-\int_{t}^{u} 2 \frac{(1-r) \pi_{r}^{n}}{\left|\widehat{\gamma}_{n}\left(\widetilde{S}_{r}^{n}\right)\right|^{2}} d r+\int_{t}^{u} \frac{(1-r)^{2}}{\left|\widehat{\gamma}_{n}\left(\widetilde{S}_{r}^{n}\right)\right|^{2}} d \pi_{r}^{n}-2 \int_{t}^{u} \frac{\pi_{r}^{n}}{(1-r)^{-2}} \frac{\left|\nabla \widehat{\gamma}_{n}\left(\widetilde{S}_{r}^{n}\right)\right|^{2}}{\left|\widehat{\gamma}_{n}\left(\widetilde{S}_{r}^{n}\right)\right|^{2}} d r-N_{u}^{Y}
\end{gathered}
$$

where $N^{Y}$ is a local martingale given by $N^{Y}:=\int_{t}^{\cdot} 2(1-r)^{2} \pi_{r}^{n} \nabla \widehat{\gamma}_{n}\left(\widetilde{S}_{r}^{n}\right) / \widehat{\gamma}_{n}\left(\widetilde{S}_{r}^{n}\right)^{2} d W_{r}$. Plugging $2 d \pi_{r}^{n}=\pi_{r}^{n} \nabla^{2} \widehat{\gamma}_{n}\left(\widetilde{S}_{r}^{n}\right) \widehat{\gamma}_{n}\left(\widetilde{S}_{r}^{n}\right) d r$ in the previous equality provides

$$
\begin{aligned}
\beta_{u}^{n} & :=\frac{1}{2} \int_{t}^{u}(1-r)^{2} \pi_{r}^{n}\left(\frac{2\left|\nabla \widehat{\gamma}_{n}\left(\widetilde{S}_{r}^{n}\right)\right|^{2}}{\left|\widehat{\gamma}_{n}\left(\widetilde{S}_{r}^{n}\right)\right|^{2}}-\frac{\nabla^{2} \widehat{\gamma}_{n}\left(\widetilde{S}_{r}^{n}\right)}{\widehat{\gamma}_{n}\left(\widetilde{S}_{r}^{n}\right)}\right) d r \\
& =\frac{(1-u)^{2} \pi_{u}^{n}}{\left|\widehat{\gamma}_{n}\left(\widetilde{S}_{u}^{n}\right)\right|^{2}}-\frac{(1-t)^{2} \pi_{t}^{n}}{\left|\widehat{\gamma}_{n}(x)\right|^{2}}+\int_{t}^{u} 2 \frac{(1-r) \pi_{r}^{n}}{\left|\widehat{\gamma}_{n}\left(\widetilde{S}_{r}^{n}\right)\right|^{2}} d r+N_{u}^{Y}, \quad t \leq u \leq 1 .
\end{aligned}
$$


Let pick $v \in[t, 1]$ and define for $r \in[v, 1], N_{r}^{*}:=\int_{v}^{r} N_{u}^{Y} d u$. By virtue of Theorem $65, I V-6,[12],\left(N_{r}^{*}\right)_{r \in[v, 1]}$ is a local martingale. Moreover, (5.62) implies that

$$
\begin{aligned}
\int_{v}^{r} \beta_{u}^{n} d u & \leq \int_{v}^{r} \frac{(1-u)^{2} \pi_{u}^{n}}{\left[\left.\widehat{\gamma}_{n}\left(\widetilde{S}_{u}^{n}\right)\right|^{2}\right.} d u+\int_{v}^{r} \int_{t}^{u} 2 \frac{(1-r) \pi_{r}^{n}}{\left|\widehat{\gamma}_{n}\left(\widetilde{S}_{r}^{n}\right)\right|^{2}} d r d u+N_{r}^{*} \\
& \leq 3(1-v) \int_{t}^{1} \frac{(1-u) \pi_{u}^{n}}{\left|\widehat{\gamma}_{n}\left(\widetilde{S}_{u}^{n}\right)\right|^{2}} d u+N_{r}^{*}
\end{aligned}
$$

Besides, observe that

$$
\int_{t}^{1} \frac{(1-r) \pi_{r}^{n}}{\left|\widehat{\gamma}_{n}\left(\widetilde{S}_{r}^{n}\right)\right|^{2}} d r=\frac{8}{\gamma_{n}^{2}} \int_{t}^{1}-(1-r) d \pi_{r}^{n} \leq \frac{8}{\gamma_{n}^{2}}(1-t) \pi_{t}^{n} .
$$

This estimate together with (5.63) and $\beta^{n} \geq 0$ imply that $\left(N_{r}^{*}\right)_{r \in[v, 1]}$ is a supermartingale, as a local martingale bounded from below. Therefore, since $\beta^{n}$ is increasing, we deduce from (5.63) and (5.64) that

$$
E \beta_{v}^{n} \leq \frac{1}{1-v} \mathbb{E} \int_{v}^{1} \beta_{u}^{n} d u \leq \frac{24}{\gamma_{n}^{2}}(1-t) \pi_{t}^{n}, \quad t \leq v<1 .
$$

As $v \rightarrow 1$, using the Fatou lemma since $\beta^{n} \geq 0$, we derive

$$
\mathbb{E} Y_{1}^{n} \leq \frac{\gamma_{n}^{2}}{4}\left|\pi_{t}^{n}\right|^{2} \mathbb{E} \beta_{1}^{n} \leq 6(1-t)\left|\pi_{t}^{n}\right|^{3}
$$

We now focus on the term $X_{1}^{n}$ and observe from (5.44) that

$$
d\left(\frac{b_{r}^{n}}{\pi_{r}^{n}}\right)=\frac{\sigma^{2} \gamma_{n}^{2} \nabla \widehat{\gamma}_{n}\left(\widetilde{S}_{r}^{n}\right)}{4\left|\widehat{\gamma}_{n}\left(\widetilde{S}_{r}^{n}\right)\right|^{2}} \pi_{r}^{n} d r=-\frac{\nabla \widehat{\gamma}_{n}\left(\widetilde{S}_{r}^{n}\right)}{2} d \pi_{r}^{n},
$$

so that $b^{n} / \pi^{n}$ is increasing and therefore $2 d\left|b_{r}^{n} / \pi_{r}^{n}\right| \leq-\nabla \widehat{\gamma}_{n}\left(\widetilde{S}_{r}^{n}\right) d \pi_{r}^{n}$. Hence, Ito's formula implies directly

$$
\begin{aligned}
X_{u}^{n} & \leq \nabla \widehat{\gamma}_{n}(x) \frac{\left|b_{t}^{n}\right|}{\pi_{t}^{n}}(1-t)\left|\pi_{t}^{n}\right|^{2}-\int_{t}^{u} \nabla \widehat{\gamma}_{n}\left(\widetilde{S}_{r}^{n}\right) \frac{\left|b_{r}^{n}\right|}{\pi_{r}^{n}}\left|\pi_{r}^{n}\right|^{2} d r \\
& -\int_{t}^{u}\left|\nabla \widehat{\gamma}_{n}\left(\widetilde{S}_{r}^{n}\right)\right|^{2}\left|\pi_{r}^{n}\right|^{2}(1-r)^{2} d \pi_{r}^{n}+\int_{t}^{u} \frac{\left|b_{r}^{n}\right|}{\pi_{r}^{n}}(1-r)\left|\pi_{r}^{n}\right|^{2} d \nabla \widehat{\gamma}_{n}\left(\widetilde{S}_{r}^{n}\right),
\end{aligned}
$$

for $t \leq u \leq 1$. Plugging (5.49) in this expression, we deduce

$$
0 \leq \frac{1}{2} X_{u}^{n} \leq \nabla \widehat{\gamma}_{n}(x)(1-t)\left|b_{t}^{n}\right| \pi_{t}^{n}+Y_{1}^{n}+N_{u}^{X}, \quad t \leq u \leq 1,
$$

where $N^{X}$ is a local martingale. Since $\mathbb{E} Y_{1}^{n}<\infty$, we deduce that $N^{X}$ is a supermartingale so that $\mathbb{E} N_{1}^{X} \leq 0$. Hence, combining (5.59) together with (5.65) and (5.66) provides

$$
\begin{aligned}
C_{3}^{n} & \leq C \sqrt{1-t}\left(\left|c_{t}^{n}\right|+\nabla \widehat{\gamma}_{n}(x)(1-t)\left|b_{t}^{n}\right| \pi_{t}^{n}+(1-t)\left|\pi_{t}^{n}\right|^{3}\right) \\
& =\frac{C}{\sqrt{1-t}}\left(\frac{\left|\nabla^{2} \widehat{\gamma}_{n}(x)\right|}{\left|\widehat{\gamma}_{n}(x)\right|^{2}}+3 \frac{\left|\nabla \widehat{\gamma}_{n}(x)\right|^{2}}{\left|\widehat{\gamma}_{n}(x)\right|^{3}}+\frac{1}{(1-t)\left|\widehat{\gamma}_{n}(x)\right|^{3}}\right) .
\end{aligned}
$$

Since $\left|\nabla^{2} \widehat{\gamma}_{n}\right| \widehat{\gamma}_{n}(x) \leq C \gamma_{n} / x, \nabla \widehat{\gamma}_{n} / \widehat{\gamma}_{n}(x) \leq C / x$ and $1 / \widehat{\gamma}_{n}(x) \leq C \sqrt{\gamma_{n} x}$, this yields

$$
C_{3}^{n} \leq \frac{C}{\gamma_{n} \sqrt{1-t}}\left(\frac{1}{x^{5 / 2}}+\frac{1}{\gamma_{n} x^{3 / 2}}\right) .
$$

Plugging (5.54), (5.57) and (5.67) in (5.51) and (5.53) provides (4.25). 
5.4 Estimate (4.26) on the crossed derivative

This subsection is dedicated to the obtention of (4.26). This finer estimate is necessary in order to consider transaction costs coefficients which do not vanish as the number of trading dates $n$ goes to infinity. It requires the obtention of stronger estimates on $\left(\widehat{C}_{x x}^{n}\right)$ and $\left(\widehat{C}_{x x x}^{n}\right)$ which are made possible via the control (3.7) on the sequence of payoff functions $\left(h^{n}\right)_{n}$.

We recall that the initial condition $(t, x)$ is fixed and $\mathbb{E}_{t, x}$ denotes $\mathbb{E}\left[. \mid \widetilde{S}_{t}^{n}=x\right]$. Let us first derive some a priori estimates on $\left(\widetilde{S}^{n}\right)_{n}$ and $\left(\nabla \widetilde{S}^{n}\right)_{n}$.

Lemma 5.1 There exist a constant $C$ and a continuous function $f$ on $(0, \infty)$ which do not depend on $n$ such that

$$
\begin{aligned}
\mathbb{E}_{t, x} \nabla \widetilde{S}_{u}^{n} & \leq C, \quad t \leq u \leq 1, \\
\mathbb{E}_{t, x} \widetilde{S}_{u}^{n} & \leq C f(x), \quad t \leq u \leq 1, \\
\mathbb{E}_{t, x}\left|\nabla \widetilde{S}_{u}^{n}\right|^{2} & \leq C f(x), \quad t \leq u \leq 1, \\
\mathbb{E}_{t, x}\left|\widetilde{S}_{u}^{n}\right|^{3 / 2} & \leq C f(x), \quad t \leq u \leq 1, \\
\mathbb{E}_{t, x}\left|\widetilde{S}_{u}^{n}\right|^{2} & \leq C \sqrt{\gamma_{n}} f(x), \quad t \leq u \leq 1,
\end{aligned}
$$

Proof. We fix $n \in \mathbb{N}$ and $u \in[t, 1]$ in order to verify each estimate separately.

Proof of (5.68).

Recall that $\nabla \widetilde{S}^{n}$ satisfies

$$
d \nabla \widetilde{S}_{u}^{n}=\nabla \widehat{\gamma}_{n}\left(\widetilde{S}_{u}^{n}\right) \nabla \widetilde{S}_{u}^{n} d W_{u}+\sigma^{2} \nabla \widetilde{S}_{u}^{n} d u
$$

Using the dynamic of $\widetilde{S}^{n}$ and the Ito formula, we verify easily that $\widetilde{S}^{n}$ has finite moments of all orders. As $\nabla \widetilde{S}_{u}^{n}=\pi_{u}^{n}(1-t) \widehat{\gamma}_{n}\left(\widetilde{S}_{u}^{n}\right)$, we deduce that $\nabla \widetilde{S}_{u}^{n}$ has also finite moments of all orders. We also know that the process $\nabla \widetilde{S}^{n}$ is positive and $\int_{t} \nabla \widehat{\gamma}_{n}\left(\widetilde{S}_{u}^{n}\right) \nabla \widetilde{S}_{u}^{n} d W_{u}$ is a local martingale which turns out to be a martingale once stopped by a sequence of stopping times $\tau^{k, n} \rightarrow \infty$ a.s. as $k \rightarrow \infty$. By the Fatou Lemma, we deduce that

$$
E \nabla \widetilde{S}_{u}^{n} \leq 1+\liminf _{k} \mathbb{E} \int_{t}^{\tau^{k, n}} \sigma^{2} \nabla \widetilde{S}_{u}^{n} d u \leq 1+\mathbb{E} \int_{t}^{u} \sigma^{2} \nabla \widetilde{S}_{r}^{n} d r .
$$

Using the Gronwall lemma, we conclude about (5.68).

Proof of (5.69).

By virtue of (5.68), we have $0 \leq \nabla_{x} \mathbb{E}_{t, x} \widetilde{S}_{u}^{n}=\mathbb{E}_{t, x} \nabla \widetilde{S}_{u}^{n} \leq C$. Hence, a Taylor expansion directly leads to

$$
\mathbb{E}_{t, x} \widetilde{S}_{u}^{n}=\mathbb{E}_{t, x} \widetilde{S}_{u}^{n}-\mathbb{E}_{t, 0} \widetilde{S}_{u}^{n} \leq C x
$$

Proof of (5.70) 
From the s.d.e. satisfied by $\widetilde{S}_{u}^{n}$, we deduce that there is a constant $C$ such that $E\left|\widetilde{S}_{u}^{n}\right|^{2} \leq C \gamma_{n} g(x)$ for some continuous function $g$. To do so, it suffices to use inequality (5.69) and apply the Gronwall lemma. Recall that

$$
\nabla \widetilde{S}^{n}=\pi^{n}(1-t) \widehat{\gamma}_{n}\left(\widetilde{S}^{n}\right)=\pi^{n}(1-t) \sqrt{\sigma^{2}\left|\widetilde{S}^{n}\right|^{2}+\sigma \gamma_{n} \widetilde{S}^{n}}
$$

As $\pi^{n} \leq \pi_{t}^{n}$, we conclude about (5.70).

Proof of (5.71).

We have $\nabla_{x} \mathbb{E}_{t, x}\left|\widetilde{S}_{u}^{n}\right|^{3 / 2}=(3 / 2) \mathbb{E}_{t, x}\left|\widetilde{S}_{u}^{n}\right|^{1 / 2} \nabla \widetilde{S}_{u}^{n}$. Using the Cauchy-Schwarz inequality and Inequalities (5.69) and (5.70), we deduce that $0 \leq \nabla_{x} \mathbb{E}_{t, x}\left|\widetilde{S}_{u}^{n}\right|^{3 / 2} \leq$ $C g(x)$, for some continuous function $g$. Hence (5.69) follows from a Taylor expansion.

\section{Proof of (5.72).}

We have $\nabla_{x} \mathbb{E}_{t, x}\left|\widetilde{S}_{u}^{n}\right|^{2}=2 \mathbb{E}_{t, x} \widetilde{S}_{u}^{n} \nabla \widetilde{S}_{u}^{n}$. We then use the Cauchy-Schwarz inequality with Inequality (5.70) and the inequality $\mathbb{E}_{t, x}\left|\widetilde{S}_{u}^{n}\right|^{2} \leq C \gamma_{n} g(x)$. The conclusion follows as previously.

We now provide finer estimates on $\left(\widehat{C}_{x x}^{n}\right)$ and $\left(\widehat{C}_{x x x}^{n}\right)$.

Lemma 5.2 There exists a continuous function $f$ such that

$$
\left|\widehat{C}_{x x}^{n}(t, x)\right| \leq \frac{f(x)}{(1-t)^{4 / 3} \gamma_{n} \ln (n)}, \quad(t, x) \in[0,1] \times(0, \infty), \quad n \in \mathbb{N} .
$$

Proof. Fix $n \in \mathbb{N}$. From (4.17) and (5.34), we compute

$\widehat{C}_{x x}^{n}(t, x)=\mathbb{E}\left[\nabla^{2} h^{n}\left(\widetilde{S}_{1}^{n}\right) \nabla \widetilde{S}_{1}^{n}\right]=\mathbb{E}\left[\nabla^{2} h^{n}\left(\widetilde{S}_{1}^{n}\right) \frac{\widehat{\gamma}_{n}\left(\widetilde{S}_{1}^{n}\right) \pi_{1}^{n}}{(1-t)^{-1}}\right] \leq \frac{C \sqrt{\gamma_{n}}\left\|\nabla^{2} h^{n}\right\|_{\infty}}{(1-t)^{-1}} \mathbb{E}\left[\pi_{1}^{n}\right]$, since $\nabla^{2} h^{n}$ vanishes outside a compact subset of $(0, \infty)$ which does not depend of $n$ and hence $\nabla^{2} h^{n}\left(\widetilde{S}_{1}^{n}\right) \widehat{\gamma}_{n}\left(\widetilde{S}_{1}^{n}\right)$ is bounded by $C \sqrt{\gamma_{n}}\left\|\nabla^{2} h^{n}\right\|_{\infty}$.

We now look towards a sharp estimate of $\mathbb{E}\left[\pi_{1}^{n}\right]$. The expression of $\pi^{n}$ given in (5.34) together with Jensen inequality yield

$$
\mathbb{E}\left[\pi_{1}^{n}\right] \leq \frac{\pi_{t}^{n}}{1-t} \int_{t}^{1} \mathbb{E} e^{-\frac{(1-t) \sigma^{2} \gamma_{n}^{2}}{8 \mid \hat{\gamma}_{n}\left(\left.\widetilde{S}_{u}^{n}\right|^{2}\right.}} d u \leq \frac{\pi_{t}^{n} \gamma_{n}^{-2}}{(1-t)^{2}} \int_{t}^{1} \mathbb{E}\left[\left|\widehat{\gamma}_{n}\left(\widetilde{S}_{u}^{n}\right)\right|^{2} e^{-\frac{(1-t) \sigma^{2} \gamma_{n}^{2}}{16\left|\hat{\gamma}_{n}\left(\widetilde{S}_{u}^{n}\right)\right|^{2}}}\right] d u,(5.73)
$$

where we used the bound $x e^{-x} \leq C, x \geq 0$, for the last inequality. We split the expectation of the r.h.s. in the expression above in two parts. The first one is bounded for $n$ large enough as follows, by virtue of (5.69) and (5.72):

$$
\mathbb{E}\left[\left|\widehat{\gamma}_{n}\left(\widetilde{S}_{u}^{n}\right)\right|^{2} e^{-\frac{(1-t) \sigma^{2} \gamma_{n}^{2}}{16 \gamma^{2}\left(\widetilde{S}_{u}^{n}\right)}} 1_{\left\{\widetilde{S}_{u}^{n} \leq \sqrt{\gamma_{n}}\right\}}\right] \leq \gamma_{n} e^{-\frac{(1-t) \sigma^{2} \gamma_{n}^{1 / 2}}{16}} f(x),
$$

where $f$ is a continuous function which may change from line to line. Observe that the Cauchy-Schwarz inequality and (5.71) yields $\mathbb{E}\left[\widetilde{S}_{u}^{n} 1_{\widetilde{S}_{u}^{n} \geq \sqrt{\gamma_{n}}}\right] \leq \gamma_{n}^{-1 / 6} f(x)$. Therefore, the second term is bounded by

$$
\mathbb{E}\left[\left|\widehat{\gamma}_{n}\left(\widetilde{S}_{u}^{n}\right)\right|^{2} e^{-\frac{(1-t) \sigma^{2} \gamma_{n}^{2}}{16 \gamma^{2}\left(\widetilde{S}_{u}^{n}\right)}} 1_{\left\{\widetilde{S}_{u}^{n} \geq \sqrt{\gamma_{n}}\right\}}\right] \leq\left(\sigma^{2} \sqrt{\gamma_{n}}+\gamma_{n}^{5 / 6}\right) f(x) \leq \gamma_{n}^{5 / 6} f(x) .
$$


Together with $x^{1 / 3} e^{-x} \leq C, x \geq 0$, plugging (5.74) and (5.75) in (5.73) yields

$$
\mathbb{E}\left[\pi_{1}^{n}\right] \leq \frac{\pi_{t}^{n} \gamma_{n}^{-2}}{(1-t)} \frac{\gamma_{n}^{5 / 6} f(x)}{(1-t)^{1 / 3}} \leq \frac{f(x)}{\gamma_{n}^{5 / 3}(1-t)^{7 / 3}} .
$$

Together with (3.7), plugging this estimate in the first inequality of this proof concludes the proof.

Lemma 5.3 Fix $n \in \mathbb{N}$. There exists a continuous function $f$ such that

$$
\left|\widehat{C}_{x x x}^{n}(t, x)\right| \leq \frac{f(x)}{(1-t)^{4 / 3} \gamma_{n} \ln (n)}, \quad(t, x) \in[0,1] \times(0, \infty), \quad n \in \mathbb{N} .
$$

Proof. Fix $n \in \mathbb{N}$. As observed in Section 5.2, we have

$\left|\widehat{C}_{x x x}^{n}(t, x)\right| \leq\left\|\nabla h^{n}\right\|_{\infty} A_{n}^{1 / 2}+\bar{B}_{n}, \quad$ where $\bar{B}_{n}:=\mathbb{E}\left[\nabla h^{n}\left(\widetilde{S}_{1}^{n}\right)\left(\int_{t}^{1} b_{u}^{n} d W_{u}\right)\right](5.7$

and $\left(A_{n}\right)_{n}$ and $\left(b^{n}\right)_{n}$ are respectively given in (5.37) and (5.40). As already observed in (5.38), we have

$A_{n} \leq \frac{(1-t)^{-2}}{\left|\widehat{\gamma}_{n}(x)\right|^{2}} \int_{t}^{1} \int_{t}^{u} \mathbb{E}\left|\pi_{s}^{n}\right|^{2} d s d u \leq \frac{(1-t)^{-2}\left|\pi_{t}^{n}\right|^{2}}{\left|\widehat{\gamma}_{n}(x)\right|^{2}} \int_{t}^{1} \int_{t}^{u} \int_{t}^{s} \frac{\mathbb{E} e^{-\frac{(s-t) \sigma^{2} \gamma_{n}^{2}}{4\left|\widehat{\gamma}^{n}\left(\tilde{S}_{r}^{n}\right)\right|^{2}}}}{s-t} d r d s d u$.

Using the bound $x^{1 / 2} e^{-x} \leq C$ for $x \geq 0$, we deduce

$$
A_{n} \leq C \frac{(1-t)^{-2}\left|\pi_{t}^{n}\right|^{2}}{\left|\widehat{\gamma}_{n}(x)\right|^{2} \gamma_{n}} \int_{t}^{1} \int_{t}^{u} \frac{1}{(s-t)^{3 / 2}} \int_{t}^{s} \mathbb{E}\left[\widehat{\gamma}_{n}\left(\widetilde{S}_{r}^{n}\right) e^{-\frac{(s-t) \sigma^{2} \gamma_{n}^{2}}{8\left|\hat{\gamma}_{n}\left(\widetilde{S}_{r}^{n}\right)\right|^{2}}}\right] d r d s d u
$$

Since the exponential on the r.h.s is smaller than 1 , we directly deduce from (5.69) that

$A_{n}^{1 / 2} \leq C \frac{(1-t)^{-1}\left|\pi_{t}^{n}\right|}{\left|\widehat{\gamma}_{n}(x)\right| \gamma_{n}^{1 / 2}} \frac{\gamma_{n}^{1 / 4} f(x)}{(1-t)^{-3 / 4}} \leq \frac{f(x)}{(1-t)^{5 / 4} \gamma_{n}^{5 / 4}} \leq \frac{f(x)}{(1-t)^{4 / 3} \gamma_{n} \ln (n)}$.

We now focus on the second term on the r.h.s. of (5.77) and rewrite

$$
\bar{B}_{n}=\mathbb{E} \int_{t}^{1} b_{u}^{n} \nabla^{2} h^{n}\left(\widetilde{S}_{1}^{n}\right) D_{u} \widetilde{S}_{1}^{n} d u=\mathbb{E} \int_{t}^{1} b_{u}^{n} \nabla^{2} h^{n}\left(\widetilde{S}_{1}^{n}\right) \frac{\nabla \widetilde{S}_{1}^{n}}{(1-t) \pi_{u}^{n}} d u, \quad t \leq u \leq 1 .
$$

Observe from (5.45) that the process $b^{n}$ is given by

$$
b_{u}^{n}=\frac{\pi_{u}^{n}}{\pi_{t}^{n}} b_{t}^{n}+\pi_{u}^{n} \int_{t}^{u} \frac{\sigma^{2} \gamma_{n}^{2} \nabla \widehat{\gamma}_{n}\left(\widetilde{S}_{r}^{n}\right)}{4 \widehat{\gamma}_{n}^{2}\left(\widetilde{S}_{r}^{n}\right)} \pi_{r}^{n}(1-r) d r .
$$

Moreover, recall that $\nabla \widetilde{S}_{1}^{n}=\pi_{1}^{n}(1-t) \widehat{\gamma}_{n}\left(\widetilde{S}_{1}^{n}\right)$ and $\nabla^{2} h^{n}$ vanishes outside a compact subset independent of $n$. Plugging these estimates in the expression of $\bar{B}_{n}$, we get $\bar{B}_{n} \leq C \sqrt{\gamma_{n}}\left\|\nabla^{2} h^{n}\right\|_{\infty}\left(\frac{\left|b_{t}^{n}\right|}{\pi_{t}^{n}}(1-t) \mathbb{E}\left[\pi_{1}^{n}\right]+\mathbb{E}\left[\pi_{1}^{n} \int_{t}^{1} \frac{\sigma^{2} \gamma_{n}^{2} \nabla \widehat{\gamma}_{n}\left(\widetilde{S}_{r}^{n}\right)}{4 \widehat{\gamma}_{n}^{2}\left(\widetilde{S}_{r}^{n}\right)} \pi_{r}^{n}(1-r)^{2} d r\right]\right)$ 
Recalling the process $\beta^{n}$ defined in (5.61), observe that the expression of $b_{t}^{n}$ together with $\left|\nabla \widehat{\gamma}_{n}\right| \leq 1+\left|\nabla \widehat{\gamma}_{n}\right|^{2}$ and (5.34) lead to

$$
\begin{aligned}
\bar{B}_{n} & \leq C \sqrt{\gamma_{n}}\left\|\nabla^{2} h^{n}\right\|_{\infty}\left(\frac{\nabla \widehat{\gamma}_{n}(x)}{\widehat{\gamma}_{n}(x)}(1-t) \mathbb{E}\left[\pi_{1}^{n}\right]+\mathbb{E}\left[\pi_{1}^{n} \int_{t}^{1}-(1-r)^{2} d \pi_{r}^{n}\right]+\mathbb{E}\left[\pi_{1}^{n} \beta_{1}^{n}\right]\right) \\
& \leq C \sqrt{\gamma_{n}}\left\|\nabla^{2} h^{n}\right\|_{\infty}\left(\frac{1-t}{x} \mathbb{E}\left[\pi_{1}^{n}\right]+(1-t)^{2} \pi_{t}^{n} \mathbb{E}\left[\pi_{1}^{n}\right]+\mathbb{E}\left[\pi_{1}^{n} \beta_{1}^{n}\right]\right) \\
& \leq \frac{\left\|\nabla^{2} h^{n}\right\|_{\infty} f(x)}{(1-t)^{4 / 3} \gamma_{n}^{7 / 6}}+C \sqrt{\gamma_{n}}\left\|\nabla^{2} h^{n}\right\|_{\infty} \mathbb{E}\left[\pi_{1}^{n} \beta_{1}^{n}\right],
\end{aligned}
$$

where the last inequality follows from (5.76).

The rest of the proof is dedicated to the control of $\mathbb{E}\left[\pi_{1}^{n} \beta_{1}^{n}\right]$. We follow the notations of the previous section and observe from the monotonicity of $\beta^{n}$ together with (5.63) that

$\mathbb{E} \pi_{1}^{n} \beta_{1}^{n} \leq \lim _{v \rightarrow 1} \mathbb{E} \pi_{1}^{n} \frac{1}{1-v} \int_{v}^{1} \beta_{u}^{n} d u \leq \lim _{v \rightarrow 1} \mathbb{E} \pi_{1}^{n}\left(3 \int_{t}^{1} \frac{(1-r) \pi_{r}^{n}}{\widehat{\gamma}_{n}^{2}\left(\widetilde{S}_{r}^{n}\right)} d r+\frac{1}{1-v} \int_{v}^{1} N_{r}^{Y} d r\right)$.

Since the first term in the parenthesis is bounded by $C \pi_{t}^{n}(1-t) \gamma_{n}^{-2},(5.76)$ yields

$$
\mathbb{E} \pi_{1}^{n} \beta_{1}^{n} \leq \frac{C \pi_{t}^{n}(1-t) \gamma_{n}^{-2}}{(1-t)^{4 / 3} \gamma_{n}^{7 / 6}} f(x)+\lim _{v \rightarrow 1} \mathbb{E} \pi_{1}^{n}\left(\frac{1}{1-v} \int_{v}^{1} N_{r}^{Y} d r\right)
$$

Regarding the last term, we first observe from (5.63) that

$$
\int_{v}^{u} N_{r}^{Y} d r \geq-3(1-v) \int_{t}^{1} \frac{(1-r) \pi_{r}^{n}}{\gamma^{2}\left(\widetilde{S}_{r}^{n}\right)} d r \geq-C(1-v) \pi_{t}^{n} \gamma_{n}^{-2}, \quad v \leq u \leq 1 .
$$

This provides an upper bound for $\left(\int_{v} N_{r}^{Y} d r\right)^{-}$and the integration by parts formula yields

$$
\pi_{u}^{n} \int_{v}^{u} N_{r}^{Y} d r \leq-C(1-v) \pi_{t}^{n} \gamma_{n}^{-2} \int_{v}^{u} d \pi_{r}^{n}+\int_{v}^{u} \pi_{r}^{n} N_{r}^{Y} d r, \quad v \leq u \leq 1
$$

Moreover, the last term on the r.h.s is a supermartingale as a bounded from below local martingale. Hence, by virtue of the Lebesgue theorem, we finally deduce that

$$
\lim _{v \rightarrow 1} \mathbb{E} \pi_{1}^{n}\left(\frac{1}{1-v} \int_{v}^{1} N_{r}^{Y} d r\right) \leq C(1-v) \pi_{t}^{n} \gamma_{n}^{-2} \lim _{v \rightarrow 1} \mathbb{E} \int_{v}^{1} d\left(\frac{\pi_{r}^{n}}{\pi_{t}^{n}}\right)=0 .
$$

Combining this estimate with (5.77), (5.78), (5.79) and (5.80) and (3.7) concludes the proof.

Proof of (4.26).

In order to derive the upper bound (4.26), it suffices to derive the expression of $\widehat{C}_{x t}^{n}(t, x)$ from $\widehat{C}_{x x}^{n}(t, x)$ and $\widehat{C}_{x x x}^{n}(t, x)$ by differentiating the p.d.e. (enn $)$ and to plug the estimates of Lemma 5.2 and Lemma 5.3 . 


\section{Appendix: proof of Proposition 3.3}

Note that we cannot immediately conclude about the existence of a solution of $\left(\mathbf{e}_{\mathbf{n}}\right)$ because the operator is not uniformly parabolic on $] 0, \infty[\otimes[0,1[$. That's why, we shall bring the problem back to another one the domain of which satisfies the required uniform parabolicity.

Fix $n \in \mathbb{N}$. By virtue of Lemma 3.1, recall that $\widehat{S}^{n}$ is the unique solution of the stochastic equation

$$
\widehat{S}_{s}^{n,(t, x)}=x+\int_{t}^{s} \widehat{\gamma}_{n}\left(\widehat{S}_{u}^{n,(t, x)}\right) d W_{u}, \quad t \leq s \leq 1, \quad(t, x) \in[0,1] \times(0, \infty),
$$

where we use the overscript $(t, x)$ in order to emphasize the initial condition. Introducing $\widehat{\gamma}_{n}^{m}: x \mapsto \sqrt{\sigma^{2} x^{2}+\sigma \gamma_{n}|x|+m^{-1}}$, we denote by $\widehat{S}^{n, m}$ the solution of

$$
\widehat{S}_{s}^{n, m,(t, x)}=x+\int_{t}^{s} \widehat{\gamma}_{n}^{m}\left(\widehat{S}_{u}^{n, m,(t, x)}\right) d W_{u}, \quad t \leq s \leq 1, \quad(t, x) \in[0,1] \times(0, \infty),
$$

for any $m>0$. Since $\left\|\widehat{\gamma}_{n}^{m}-\widehat{\gamma}_{n}\right\|_{\infty} \leq m^{-1 / 2}$, for $m>0$, hence $\widehat{S}_{1}^{n, m,(t, x)} \rightarrow \widehat{S}_{1}^{n,(t, x)}$ in $L^{2}(\Omega, P)$ as $m$ goes to $\infty$, uniformly in $(t, x) \in[0,1 \times(0, \infty)$. We deduce that $\widehat{C}^{n, m}:(t, x) \mapsto \mathbb{E}_{t} h^{n}\left(\widehat{S}_{1}^{n, m,(t, x)}\right)$ converges uniformly to $\widehat{C}^{n}:(t, x) \mapsto \mathbb{E}_{t} h^{n}\left(\widehat{S}_{1}^{n,(t, x)}\right)$.

Applying Lemma 3.3 p 112 with Condition $\left(A^{\prime}\right)$ p 113 [3], implies, together with $\left|\nabla h^{n}\right| \leq L$, that

$$
\left|\widehat{C}^{n, m}(t, x)-\widehat{C}^{n, m}(u, y)\right| \leq L \sqrt{\mathbb{E}\left|\widehat{S}_{1}^{n,(x, t)}-\widehat{S}_{1}^{n,(u, y)}\right|^{2}} \leq K \sqrt{(x-y)^{2}+|t-u|}
$$

for $m>0,0 \leq t, u \leq 1$ and $x, y \leq|R|$ for a given $R \in(0, \infty)$, where the constant $K$ depends on $n, m$ and $R$. We deduce that $\widehat{C}^{n, m}$ is continuous for any $m>0$ and hence so is $\widehat{C}^{n}$.

Fix $m>0$. We use arguments of Section 6.3 in [3] and try to follow their notations. Let us consider the following sets

$$
\begin{aligned}
Q_{m}:=(0,1) \times\left(\frac{1}{m}, m\right), & B_{m}:=\{1\} \times\left(\frac{1}{m}, m\right), \\
T_{m} & :=\{0\} \times\left(\frac{1}{m}, m\right), \quad S_{m}:=[0,1) \times\left\{\frac{1}{m}, m\right\},
\end{aligned}
$$

For each $y \in S_{m}$, it is easy to observe that there exists a closed ball $K_{y}^{m}$ such that $K_{y}^{m} \cap Q_{m}=\emptyset$ and $K_{y}^{m} \cap \overline{Q_{m}}=\{y\}$. It follows that the function $W_{y}$ proposed $\mathrm{p}$ 134 [3] defines a barrier for each $y \in S_{m}$. Besides, $\widehat{C}^{n}$ and $h^{n}$ are continuous and $\widehat{\sigma}_{n}$ is Lipschitz on $\overline{Q_{m}}$. By virtue of Theorem $3.6 \mathrm{p} 138$ [3], we deduce that the Dirichlet problem

$$
\left\{\begin{array}{lc}
u_{t}(t, x)+\frac{1}{2} \widehat{\sigma}_{n}^{2}(x) x^{2} u_{x x}(t, x)=0 & (t, x) \in Q_{m} \cup T_{m} \\
u(T, x)=h^{n}(x) & x \in B_{m} \\
u(t, x)=\widehat{C}^{n}(t, x) & (t, x) \in S_{m}
\end{array}\right.
$$


admits a unique solution $u^{n, m}$, continuous on $\overline{Q_{m}}$ with continuous derivatives $u_{t}^{n, m}, u_{x x}^{n, m}$ on $Q_{m} \cup T_{m}$. Moreover, Theorem $5.2 \mathrm{p} 147$ [3] implies that $u^{n, m}$ has the following stochastic representation

$$
u^{n, m}(t, x)=\mathbb{E}\left[\widehat{C}^{n}\left(\tau^{m}, \widehat{S}_{\tau^{m}}^{n,(t, x)}\right) \mathbf{1}_{\tau^{m}<1}+h^{n}\left(\widehat{S}_{1}^{n(t, x)}\right) \mathbf{1}_{\tau^{m}=1}\right], \quad(t, x) \in Q_{m},
$$

where $\tau^{m}$ is the first time where $\widehat{S}^{n,(t, x)}$ exits $Q_{m}$. The definition of $\widehat{C}^{n}$ implies

$$
u^{n, m}(t, x)=\mathbb{E}\left[\widehat{C}^{n}\left(\tau^{m}, \widehat{S}_{\tau^{m}}^{n,(t, x)}\right)\right]=\mathbb{E}\left[h^{n}\left(\widehat{S}_{1}^{n(t, x)}\right)\right]=\widehat{C}^{n}(t, x), \quad(t, x) \in Q_{m} .
$$

As $m \rightarrow \infty$, we deduce that $\widehat{C}^{n}$ solves the PDE (en). Moreover, $\bar{C}^{n}:(t, y) \mapsto$ $\widehat{C}^{n}\left(t, e^{y}\right)$ solves the following uniformly parabolic PDE

$$
\left\{\begin{array}{ll}
v_{t}(t, y)+\frac{1}{2} \widehat{\sigma}_{n}^{2}\left(e^{y}\right) v_{y y}(t, y)-\frac{1}{2} \widehat{\sigma}_{n}^{2}\left(e^{y}\right) v_{y}(t, y)=0, & (t, y) \in[0,1) \times \mathbb{R} \\
v(1, y)=h\left(e^{y}\right), & y \in \mathbb{R}
\end{array} .\right.
$$

By virtue of Theorem 3.6 [3], $\bar{C}^{n}$ is also the unique solution of the same PDE restricted to an arbitrary smooth bounded domain. Moreover, Theorem $5.2 \mathrm{p} 147$ [3], implies that $\bar{C}^{n}$ has a unique probabilistic representation. We deduce that $\widehat{C}^{n}$ is the unique solution of $\left(\mathbf{e}_{\mathbf{n}}\right)$.

\section{References}

1. Cherny A.S. and Engelbert H.J. Singular Stochastic Differential Equations. Lecture Notes in Mathematics. Springer.

2. Denis E., Yuri Kabanov. Mean Square Error for the Leland-Lott Hedging Strategy: Convex Pay-off. Finance and Stochastics, 14, 4, 625-667, (2010).

3. Friedman A. Stochastic Differential Equations and Applications. Volume 1. Academic Press, (1975).

4. Fournié E., Lasry J.M., Lebuchoux J., Lions P.L., Touzi N. Applications of Malliavin Calculus to Monte Carlo Methods in Finance, Finance and Stochastics 3, 391-412, (1999).

5. Fukasawa, M. (2011). Conservative delta hedging under transaction costs, Preprint.

6. Kabanov Y., Safarian M. On Leland's strategy of Option Pricing with Transaction Costs. Finance and Stochastics, 1, 3, 239-250, (1997).

7. Karatzas.I, Shreve.S.E. Brownian Motion and Stochastic Calculus. Springer Verlag.

8. Leland H. Option pricing and Replication with Transactions Costs, Journal of Finance, XL, 5, 1283-130, (1985).

9. Lépinette E. Modified Leland's Strategy for Constant Transaction Costs Rate. Mathematical Finance (2012). doi: 10.1111/j.1467-9965.2011.00498.x. To appear.

10. Lott K. Ein Verfahren zur Replikation von Optionen unter Transaktionkosten in stetiger Zeit, Dissertation. Universität der Bundeswehr München. Institut für Mathematik und Datenverarbeitung, (1993).

11. Pergamenchtikov S. Limit Theorem for Leland's Strategy. Annals of Applied Probability, 13, 1099-1118, (2003).

12. Protter P.E. Stochastic Integration and Differential Equations. 2nd. Ed. 2.1 Stochastic Modelling and Applied Probability. Springer.

13. Sekine J., Yano J. Hedging Errors of Leland's Strategies with time-inhomogeneous Rebalancing. Preprint. 